\title{
Necessity of Vanishing Shadow Price in Infinite Horizon Control Problems
}

\author{
Dmitry Khlopin
}

Received: 10 August 2011 / Revised: 29 July 2013 /

Published online: 10 October 2013

(C) Springer Science+Business Media New York 2013

\begin{abstract}
This paper refines the necessary optimality conditions for uniformly overtaking optimal control on infinite horizon in the free end case. This condition is applicable to general non-stationary systems and the optimal objective value is not necessarily finite. In the papers of S.M. Aseev, A.V. Kryazhimskii, V.M. Veliov, K.O. Besov there was suggested a boundary condition for equations of the Pontryagin Maximum Principle. Each optimal process corresponds to a unique solution satisfying the boundary condition. Following A. Seierstad's idea, in this paper we prove a more general geometric version of that boundary condition. We show that this condition is necessary for uniformly overtaking optimal control on infinite horizon in the free end case. A number of assumptions under which this condition selects a unique Lagrange multiplier is obtained. Some examples are discussed.
\end{abstract}

Keywords Optimal control - Infinite horizon problem - Transversality condition for infinity • Necessary conditions • Uniformly overtaking optimal control • Shadow price $\cdot$ Unique Lagrange multiplier

Mathematics Subject Classifications (2010) $49 \mathrm{~K} 15 \cdot 49 \mathrm{~J} 45 \cdot 37 \mathrm{~N} 40 \cdot 91 \mathrm{~B} 62$

\footnotetext{
D. Khlopin $(\bowtie)$

Krasovskii Institute of Mathematics and Mechanics, Ural Branch, Russian Academy of Sciences,

16, S. Kovalevskaja St., 620990, Yekaterinburg, Russia

e-mail: khlopin@imm.uran.ru,glukanat@mail.ru

D. Khlopin

Department of Applied Mathematics, Institute of Mathematics and Computer Science, Ural Federal University, 4, Turgeneva St., 620083,

Yekaterinburg, Russia
} 


\section{Introduction}

The Pontryagin Maximum Principle for infinite horizon problems had already been formulated in monograph [34]; the general Maximum Principle for infinite interval was proved in [23], but such Maximum Principle has no transversality condition and, in general, selects a much too broad family of extremal trajectories. A significant number $[3,9,11,23,25,32,38,40]$ of such conditions was proposed; however, as it was noted in [23, 32, 37, 39], [3, Section 6], [36, Example 10.2], these conditions may fail; even if they do hold, these conditions may fail to give any information on determining a solution of the adjoint equation.

Since the necessity of this condition does not imply its nontriviality on solutions of relations of the Maximum Principle, it is reasonable to search for a condition that would select a single solution of relations of the Maximum Principle for each optimal control. For this purpose, [36] proposes to find $\psi^{0}$ such that it is a pointwise limit of a sequence of shadow prices that equal zero on certain sequence of times. Under assumptions of [36, Theorem 6.1], such $\psi^{0}$ is unique; in what follows, it will be referred to as $\tau$-vanishing shadow price.

In papers [1-4], Aseev and Kryazhimskii proposed an explicit expression for the shadow prices. This version of the normal form of the Maximum Principle holds with the explicitly specified shadow price. This gives a complete set of necessary optimality conditions (see [1-4]); moreover, under assumptions of [5, 7-9, 36], the solution of this form of the Maximum Principle is uniquely determined by the optimal control.

This paper aims to merge these two approaches, to find assumptions such that a $\tau$-vanishing Lagrange multiplier of the Maximum Principle corresponds to each optimal control, and to express its shadow price explicitly in the form of an improper integral that depends only on optimal control and trajectory.

In this paper, we consider only the problem with free right end. It is assumed a priori that a uniformly weakly overtaking optimal control exists (for discussion of existence refer to $[10,12-14,19,26,49])$. In addition to this, all functions are assumed to be smooth in $x$. We also do not concern ourselves with sufficient optimality conditions (see [3, Section 13], [12, 35, 37, 38, 42]).

The structure of the paper is as follows. We begin with formulating the general control problem and stating general notation and main assumptions (Section 2). Then, we formulate certain useful propositions from topology and stability theory (Section 3). After that we discuss relations of the Maximum Principle and introduce the notion of $\tau$-vanishing Lagrange multipliers. Then we show that its existence is a necessary optimality condition (Theorem 2). Connection between $\tau$-vanishing Lagrange multiplier and degenerate problems is investigated in Section 5.2; for discussion of the condition $\psi^{0}(t) \rightarrow 0$, refer to Section 5.1. The problems with monotonic right-hand side are investigated in Section 5.3. Section 6 is mainly aimed at obtaining the most diverse sets of conditions under which a $\tau$-vanishing shadow price can be explicitly expressed by a Cauchy-type formula. There we also discuss connections with the results of $[1,3,5,7,8,36]$.

The last section is completely devoted to analysis of examples. We show how the choice of a sequence of $\tau$ from a number of uniformly weakly optimal solutions selects what is needed most with the help of $\tau$-vanishing shadow price (Example 2). Example 3 demonstrates that finding the $\tau$-vanishing Lagrange multiplier allows us 
to solve abnormal problems in almost the same way as normal problems are solved. Example 4 shows that even if a non-degenerate multiplier is unique, it does not necessarily satisfy weak transversality condition (20b) or has explicit representation (22c) (understood in the sense of $[1-5,7,8]$ ). In Example 5, the search for an optimal solution is reduced to a boundary value problem.

A part of results of this paper was announced in $[28,29]$. The case of $\tau$-strong optimal control was considered in [30]. A modification of Theorem 2 was published in [31].

\section{Preliminaries}

We consider the time interval $\mathbb{T} \triangleq \mathbb{R}_{\geq 0}$. The phase space of our control system is the finite-dimensional metric space $\mathbb{X} \triangleq \mathbb{R}^{m}$; denote the unit ball in $\mathbb{X}$ by $\mathbb{D}$. Denote by $\mathbb{L}$ the linear space of all real $m \times m$ matrices; equip $\mathbb{L}$ with the operator norm. The symbol $E$ (which may be equipped with some indices) denotes finite-dimensional Euclidean spaces.

Here and below, for all $t \in \mathbb{T}$, for each integrable function $a$ of time, the integral $\int_{t}^{\infty} a(\vartheta) d \vartheta$ is the limit $\int_{t}^{T} a(\vartheta) d \vartheta$ as $T \rightarrow \infty$.

Let $C(T, E)$ be a topological space of all continuous functions of $T$ to $E$; let us equip this space with the extended norm $\|\cdot\|_{C}$ of uniform convergence. Also, let topological space $C_{l o c}(T, E)$ be the set $C(T, E)$ equipped with the compact-open topology.

Let us also consider a finite-dimensional Euclidean space $U$ and map $U$ from $T$ to the set of all subsets of $\mathbb{U}$. The set $\mathfrak{U}$ of admissible controls is understood as the set of all Borel measurable locally bounded selectors of the multi-valued map $U$. The topology on $\mathfrak{U}$ is defined through the inclusion $\mathfrak{U} \subset \mathcal{L}_{\text {loc }}^{1}(\mathbb{T}, \mathbb{U})$.

A function $a: \mathbb{T} \times E_{1} \times \mathbb{U} \rightarrow E_{2}$ is said to

(1) satisfy the Carathéodory conditions if (a) the function $a(\cdot, x, u): \mathbb{T} \rightarrow E_{2}$ is Borel measurable for all $(x, u) \in E_{1} \times \mathbb{U}$, (b) the function $a(t, \cdot, \cdot): E_{1} \times \mathbb{U} \rightarrow$ $E_{2}$ is continuous for a.a. $t \in \mathbb{T}$.

(2) be locally Lipshitz continuous if for each compact subset $K$ of $E_{1} \times \mathbb{U}$ there exists a function $L_{K}^{a} \in \mathcal{L}_{l o c}^{1}(\mathbb{T}, \mathbb{T})$ satisfying $\|a(t, x, u)-a(t, y, u)\|_{E_{2}} \leq L_{K}^{a}(t) \| x-$ $y \|_{E_{1}}$ for all $(x, u),(y, u) \in K, t \in \mathbb{T}$.

(3) be integrally bounded (on each compact subset) if for each compact subset $K$ of $E_{1} \times \mathbb{U}$ there exists a function $M_{K}^{a} \in \mathcal{L}_{l o c}^{1}(\mathbb{T}, \mathbb{T})$ satisfying $\|a(t, x, u)\|_{E^{\prime \prime}} \leq$ $M_{K}^{a}(t)$ for all $(x, u) \in K, t \in \mathbb{T}$.

We assume the following conditions hold:

Condition (u): $\quad U$ is a compact-valued map, and its graph is Borel set.

Condition (fg): Locally Lipshitz continuous on $x$ Carathéodory functions $f: \mathbb{T} \times$ $\mathbb{X} \times \mathbb{U} \rightarrow \mathbb{X}, g: \mathbb{T} \times \mathbb{X} \times \mathbb{U} \rightarrow \mathbb{R}, \quad \frac{\partial f}{\partial x}: \mathbb{T} \times \mathbb{X} \times \mathbb{U} \rightarrow \mathbb{L}, \quad \frac{\partial g}{\partial x}: \mathbb{T} \times$ $\mathbb{X} \times \mathbb{U} \rightarrow \mathbb{X}$ are integrally bounded (on each compact subset); in addition, $f$ satisfies the sublinear growth condition.

Let us consider the control system

$$
\dot{x}=f(t, x, u), x(0)=x_{* *}, \quad t \in \mathbb{T}, x \in \mathbb{X}, u(t) \in U(t),
$$


where $x_{* *} \in \mathbb{X}$ is an initial value. Now we can assign the solution of (1a) to each $u \in \mathfrak{U}$. The solution is unique and it can be extended to the whole $\mathbb{T}$. Let us denote it by $x^{u}$. The map $u \mapsto x^{u}$ of $\mathfrak{U}$ to $C_{\text {loc }}(\mathbb{T}, \mathbb{X})$ is continuous [43].

In what follows, we study the problem of maximizing the objective integral functional

$$
J^{u}(T) \stackrel{T \rightarrow \infty}{\sim} \max ; \quad J^{u}(T) \triangleq \int_{0}^{T} g\left(t, x^{u}(t), u(t)\right) d t .
$$

If there is no limit in (1b), the optimality may be defined in diverse ways (for details, see $[12,14,41,42])$; generally, we will use the following definition:

Definition 1 We say that a control $u^{0} \in \mathfrak{U}$ is weakly uniformly overtaking optimal (see [13]) if

$$
\limsup _{t \rightarrow \infty} \sup _{u \in \mathfrak{U}}\left(J^{u}(t)-J^{u^{0}}(t)\right)=0 .
$$

For every sequence $\tau \triangleq\left(\tau_{n}\right)_{n \in \mathbb{N}} \uparrow \infty$ of times, we say that a control $u^{0} \in \mathfrak{U}$ is $\tau$ optimal if

$$
\limsup _{n \rightarrow \infty} \sup _{u \in \mathfrak{U}}\left(J^{u}\left(\tau_{n}\right)-J^{u^{0}}\left(\tau_{n}\right)\right)=0 .
$$

We also assume:

Condition $(\tau)$ : There exists a weakly uniformly overtaking optimal control $u^{0} \in \mathfrak{U}$ for problem (1a)-(1b).

The assumptions of existence of such control will not be studied here; many existence results for optimal solutions over infinite horizon are collected in [49].

By this condition there exist an unbounded sequence $\tau \uparrow \infty$ and some sequence $\left(\gamma_{n}\right)_{n \in \mathbb{N}} \in \mathbb{T}^{\mathbb{N}}$, converging to zero, such that

$$
J^{u^{0}}\left(\tau_{n}\right) \geq J^{u}\left(\tau_{n}\right)-\gamma_{n}^{2} \quad \forall u \in \mathfrak{U}, n \in \mathbb{N} .
$$

Then, the control $u^{0}$ is $\tau$-optimal. Fix a sequence $\tau$. Denote by $x^{0}$ the trajectory that corresponds to $u^{0}$.

Thus, any weakly uniformly overtaking optimal control is $\tau$-optimal for some sequence $\tau \uparrow \infty$. Similarly, every uniformly overtaking [13, 24] optimal control is $\tau$-optimal for every sequence $\tau \uparrow \infty$. Since the definition of $\tau$-optimality refines these definitions, it is especially convenient if such sequence $\tau$ is given initially.

Slightly simplifying the notation when passing from a sequence $\tau \triangleq\left(\tau_{n}\right)_{n \in \mathbb{N}}$ to its subsequence $\tau^{\prime}$, we will plainly write "subsequence $\tau^{\prime} \subset \tau$ ".

\section{Auxiliary Results}

\subsection{The Set $\widetilde{\mathfrak{U}}$ of Generalized Controls}

For each $u \in \mathbb{U}$, the symbol $\tilde{\delta}(u)$ denotes the probability measure concentrated at the point $u$. Denote by $\widetilde{\mathfrak{U}}_{n}$ the family of all weakly measurable mappings $\eta$ of $[0, n]$ 
to the set of Radon probability measures over $\mathbb{U}$ such that $\eta(U(t))=1$ for a.a. $t \in[0, n]$. Let us equip this set with the topology of *-weak convergence. Then, the obtained topological space is a compact [44, IV.3.11], and the set $\mathfrak{U}_{n} \triangleq\left\{\tilde{\sigma}_{[0, n]} \mid u \in \mathfrak{U}\right\}$ is everywhere densely included in $\widetilde{\mathfrak{U}}_{n}[44$, IV.3.10] by the map $u \rightarrow \widetilde{\delta} \circ u$. We also keep the notation $\widetilde{u}^{0} \triangleq \widetilde{\delta} \circ u^{0}$.

Now, let us introduce the set of all maps $\eta$ of $\mathbb{T}$ into the set of Radon probability measures over $\mathbb{U}$ such that $\left.\eta\right|_{[0, n]} \in \widetilde{\mathfrak{U}}_{n}$ for every $n \in \mathbb{N}$, and let us denote it by $\widetilde{\mathfrak{U}}$. For every $n \in \mathbb{N}$, let the projections $\tilde{\pi}_{n}: \tilde{\mathfrak{U}} \rightarrow \tilde{\mathfrak{U}}_{n}$ be given by $\left.\tilde{\pi}_{n}(\eta) \triangleq \eta\right|_{[0, n]}$ for all $\eta \in \widetilde{\mathfrak{U}}$. Let us equip $\widetilde{\mathfrak{U}}$ with the weakest topology such that all projections are continuous. The set $\mathfrak{U}$ is called the set of generalized controls.

Let us assume that for the Euclidean space $E$, the function $a: \mathbb{T} \times E \times \mathbb{U} \rightarrow E$ is a locally Lipshitz continuous integrally bounded Carathéodory function that satisfies the extendability condition on $\mathbb{T}$ (for example, if the sublinear growth condition holds; see $[43,1.4 .3])$.

Let us fix a set $\Xi \subset E$ of initial values and the system for $u \in \mathfrak{U}$ :

$$
\dot{y}=a(t, y(t), u(t)), y(0)=\xi \in \Xi, \quad t \in \mathbb{T}, u \in \mathfrak{U} .
$$

It can also be generalized for $\eta \in \widetilde{\mathfrak{U}}$ :

$$
\dot{y}=\int_{U(t)} a(t, y(t), u) d \eta(t), y(0) \in \Xi, \quad t \in \mathbb{T}, \eta \in \widetilde{\mathfrak{U}} .
$$

Each its local solution can be extended onto the whole $\mathbb{T}$. For every $\eta \in \widetilde{\mathfrak{U}}$, let us denote the family of all solutions $y \in C_{l o c}(\mathbb{T}, E)$ of system (3b) by $\widetilde{\mathfrak{A}}[\eta]$. Such transition from a system defined for $u \in \mathfrak{U}$ (like (3a)) to a generalized system, which is defined for $\eta \in \widetilde{U}$ (like (3b)), will be done sufficiently often. To avoid writing the generalized relation, we will write the initial one with the sign " $\because$." For example, we will write $(3 \mathrm{a})$ instead of (3b). In particular, for a solution $x^{\eta} \in C_{\text {loc }}(\mathbb{T}, \mathbb{X})$ of the Cauchy problem $(\widetilde{1 a})$, the function $T \mapsto \widetilde{J}^{\eta}(T)$ could by introduced, for every $\eta \in \widetilde{\mathfrak{U}}$, by the rule $(\widetilde{1 b})$.

\section{Proposition 1 [31, Proposition 6.1] Assume (u). Then,}

(1) the space $\widetilde{\mathfrak{U}}$ is a metrizable compact, and $\tilde{\delta}(\mathfrak{U})$ is everywhere dense in it;

(2) the map $\widetilde{\mathfrak{A}}: \widetilde{\mathfrak{U}} \rightarrow C_{\text {loc }}(\mathbb{T}, E)$ is continuous and $\widetilde{\mathfrak{A}}[\widetilde{\delta} \circ \mathfrak{U}]$ of admissible trajectories is everywhere dense in a compact $\widetilde{\mathfrak{A}}[\widetilde{\mathfrak{U}}] \subset C_{l o c}(\mathbb{T}, E)$ of generalized trajectories for each compact $\Xi \subset E$ of initial values;

(3) If (fg) holds, then the map $\eta \mapsto x^{\eta}$ of $\widetilde{\mathfrak{U}}$ to $C_{\text {loc }}(\mathbb{T}, \mathbb{X})$ and the map $\eta \mapsto \widetilde{J}^{\eta}$ of $\widetilde{\mathfrak{U}}$ to $C_{\text {loc }}(\mathbb{T}, \mathbb{R})$ are continuous.

Let us also note that embedding of the initial space $\mathfrak{U}$ of admissible controls into a space with a more convenient topology is a well-known trick; see, for example, [22, 44], and [13, 17, 18], [3, Section 8] for infinite horizon problems. A weak compactness was used, for example, in $[10,14,19]$. For the games on infinite horizon there is a more general construction than the one we consider here; the construction for generalized open-loop controls was studied by the author in [27]. 
3.2 Stability and Thin Tubes of Solutions

Let $w: \mathbb{T} \times \mathbb{U} \rightarrow \mathbb{T}$ be an integrally bounded (on each compact subset) Carathéodory map. For all $\tau \in \mathbb{T}$ and $\eta \in \widetilde{U}$, let us introduce

$$
\mathfrak{L}_{w}[\eta](\tau) \triangleq \int_{0}^{\tau} \int_{U(t)} w(t, u) d \eta(t) d t .
$$

Let us assume that for every $\eta \in \widetilde{\mathfrak{U}}$ from $\mathfrak{L}_{w}[\eta](\tau)=0$ for all $\tau \in \mathbb{T}$ it follows that $\eta$ equals $\widetilde{u}^{0}$ a.e. on $[0, \tau]$. The set of such $w$ is denoted by $(N u l l)\left(u^{0}\right)$. Note that $\mathfrak{L}_{w}\left[\widetilde{u}^{0}\right] \equiv$ 0 for all $w \in(N u l l)\left(u^{0}\right)$.

For every position $\left(\vartheta^{*}, y^{*}\right) \in \mathbb{T} \times E$, there exists a unique solution $y \in C(\mathbb{T}, E)$ of the equation

$$
\dot{y}=a\left(t, y(t), u^{0}(t)\right), \quad y\left(\vartheta^{*}\right)=y^{*} .
$$

The solution continuously depends on $\left(\vartheta^{*}, y^{*}\right)$. Let us denote its initial position $y(0)$ by $\varkappa\left(\vartheta^{*}, y^{*}\right)$.

Proposition 2 Let $\Xi$ be a compact subset of $E$.

Then, there exists $w^{0} \in(N u l l)\left(u^{0}\right)$ such that for arbitrary $\eta \in \widetilde{\mathfrak{U}}, T \in \mathbb{T}$ for every solution y of (3b) from $\varkappa(\vartheta, y(\vartheta)) \in \Xi$ for all $\vartheta \in[0, T]$ it follows that

$$
\|\varkappa(\vartheta, y(\vartheta))-y(0)\|_{E} \leq \mathfrak{L}_{w^{0}}[\eta](\vartheta) \quad \forall \vartheta \in[0, T] .
$$

In the geometric sense, this proposition means that if a solution $\left.y\right|_{[0, T]}$ from the funnel $\widetilde{\mathfrak{A}}[\eta]$ does not escape the area $\widetilde{\mathfrak{A}}\left[u^{0}\right]$, then it also does not escape the tube of solutions of (3c), breadth of which (at $t=0$ ) does not surpass $\mathfrak{L}_{w^{0}}[\eta](T)$. See the proof in Appendix.

\section{$4 \tau$-Vanishing Lagrange Multiplier as a Necessary Condition}

\subsection{Core Relations of the Maximum Principle}

In what follows, we consider the shadow price $\psi$ a covector (a row vector); however, we will still write $x \in \mathbb{X}, \psi \in \mathbb{X}$ and will not distinguish between the space $\mathbb{X}$ and its conjugate space in the sense of sets.

Let the Hamilton-Pontryagin function $\mathcal{H}: \mathbb{X} \times \mathbb{T} \times \mathbb{U} \times \mathbb{T} \times \mathbb{X} \rightarrow \mathbb{R}$ be given by

$$
\mathcal{H}(x, t, u, \lambda, \psi) \triangleq \psi f(t, x, u)+\lambda g(t, x, u) .
$$

Let us introduce the relations and boundary condition:

$$
\begin{aligned}
\dot{x}(t) & =f(t, x(t), u(t)) ; \\
\dot{\psi}(t) & =-\frac{\partial \mathcal{H}}{\partial x}(x(t), t, u(t), \lambda, \psi(t)) ; \\
\sup _{p \in U(t)} \mathcal{H}(x(t), t, p, \lambda, \psi(t)) & =\mathcal{H}(x(t), t, u(t), \lambda, \psi(t)) ;
\end{aligned}
$$




$$
x(0)=x_{* *}, \quad\|\psi(0)\|_{\mathbb{X}}+\lambda=1 .
$$

It is easily seen that, for each $u \in \mathfrak{U}$ for each initial condition, system (4a), (4b) has a local solution, and each solution of these relations can be extended to the whole $\mathbb{T}$. Let us denote by $\mathfrak{Y}$ the family of all solutions $(x, u, \lambda, \psi) \in C_{l o c}(\mathbb{T}, \mathbb{X}) \times \mathfrak{U} \times[0,1] \times$ $C_{\text {loc }}(\mathbb{T}, \mathbb{X})$ of system $(4 a),(4 b),(5 a)$ on $\mathbb{T}$. Let us denote by $\mathfrak{Z}$ the set of solutions from $\mathfrak{Y}$ such that $(4 c)$ also holds a.e. on $\mathbb{T}$.

Let us embed the sets $\mathfrak{Y}$ and $\mathfrak{Z}$ into $C_{\text {loc }}(\mathbb{T}, \mathbb{X}) \times \widetilde{\mathfrak{U}} \times[0,1] \times C_{\text {loc }}(\mathbb{T}, \mathbb{X})$ by the mapping $(I d, \widetilde{\delta}, I d, I d)$; denote closures of their images by $\widetilde{\mathfrak{Y}}$ and $\widetilde{\mathfrak{Z}}$, respectively; then, $\widetilde{\mathfrak{Y}}$ and $\widetilde{\mathfrak{Z}}$ are compacts.

By Proposition 1 , for every $(x, \eta, \lambda, \psi) \in \widetilde{\mathfrak{Y}}$, the following relations hold: (5a), $\widetilde{(4 \mathrm{a})}-(\widetilde{4 b})$; for $(x, \eta, \lambda, \psi) \in \widetilde{\mathfrak{Y}}$, we also have $\widetilde{4 \mathrm{c})}$, i.e.,

$$
\sup _{p \in U(t)} \mathcal{H}(x(t), t, p, \lambda, \psi(t))=\int_{U(t)} \mathcal{H}(x(t), t, u, \lambda, \psi(t)) d \eta(t) .
$$

Moreover, Proposition 1 implies that all solutions of these equations depend on both controls $u \in \widetilde{\mathfrak{U}}$ and initial conditions continuously on any compact.

A nontrivial Lagrange multiplier $(\lambda, \psi) \in[0,1] \times C_{l o c}(\mathbb{T}, \mathbb{X})$ is called a Lagrange multiplier associated with $\left(x^{0}, u^{0}\right)$ if $\left(x^{0}, u^{0}, \lambda, \psi\right)$ is a solution of core relations of Maximum Principle, i.e. the system (4a)-(4c). It is convenient to denote by $\Lambda$ the family of all Lagrange multipliers $(\lambda, \psi) \in\{0,1\} \times C_{l o c}(\mathbb{T}, \mathbb{X})$ associated with $\left(x^{0}, u^{0}\right)$ such that

$$
\lambda=1 \text { or } \quad\left(\lambda=0 \text { and }\|\psi(0)\|_{\mathbb{X}}=1\right) .
$$

For each $\xi \in \mathbb{X}$, let us also define solutions $x_{\xi} \in C(\mathbb{T}, \mathbb{X}), A_{\xi} \in C(\mathbb{T}, \mathbb{L})$ of the following equations:

$$
\begin{aligned}
\dot{x}_{\xi}(t) & =f\left(t, x_{\xi}(t), u^{0}(t)\right) \quad x_{\xi}(0)=x_{* *}+\xi \\
\dot{A}_{\xi}(t) & =\frac{\partial f}{\partial x}\left(t, x_{\xi}(t), u^{0}(t)\right) A_{\xi}(t) \quad A_{\xi}(0)=1_{\mathbb{L}} .
\end{aligned}
$$

For every $T \in \mathbb{T}$, consider the covector

$$
I_{\xi}(T) \triangleq \int_{0}^{T} \frac{\partial g}{\partial x}\left(t, x_{\xi}(t), u^{0}(t)\right) A_{\xi}(t) d t .
$$

Similarly, for each $u \in \mathfrak{U}$, let us introduce a matrix function $A^{u}$ and a covector function $I^{u}$ by the relations

$$
\begin{gathered}
\dot{A}^{u}(t)=\frac{\partial f}{\partial x}\left(t, x^{u}(t), u(t)\right) A^{u}(t), \quad A^{u}(0)=1_{\mathbb{L}}, \\
I^{u}(T) \triangleq \int_{0}^{T} \frac{\partial g}{\partial x}\left(t, x^{u}(t), u(t)\right) A^{u}(t) d t \quad \forall T \in \mathbb{T} .
\end{gathered}
$$

In addition, we call $x^{\eta}, A^{\eta}, \psi^{\eta}, I^{\eta}, J^{\eta}$ the solutions of the corresponding -equations, or, equivalently, the limits, uniform on compacts, of $x^{u}, A^{u}, \psi^{u}, I^{u}, J^{u}$ as $\tilde{\delta}(u) \rightarrow \eta$ in the $*$-weak topology of $\widetilde{\mathfrak{U}}$. 
Expressing the solution of linear equation (4b) through (6c) (or (6b)), then any shadow price $\psi$ has the form

$$
\psi(T)=(\psi(0)-\lambda I(T)) A^{-1}(T) \quad \forall T \in \mathbb{T} ;
$$

and we can reformulate the result of [23] in the following way:

Theorem 1 [23] Under conditions (u), (fg), for any $\tau$-optimal pair $\left(x^{0}, u^{0}\right) \in$ $C_{\text {loc }}(\mathbb{T}, \mathbb{X}) \times \mathfrak{U}$ of problem (1a)-(1b), for some $\lambda^{0} \in[0,1], \psi^{0} \in C(\mathbb{T}, \mathbb{X})$, core relations of the Maximum Principle (4a)-(5a) hold for $\left(x^{0}, u^{0}, \lambda^{0}, \psi^{0}\right)$, i.e., $\left(x^{0}, u^{0}, \lambda^{0}, \psi^{0}\right) \in \mathfrak{Z}$.

Moreover, up to a positive factor, for some $I_{*} \in \mathbb{X}, \iota_{*} \in \mathbb{X}$, one of the two following relations also holds:

$$
\begin{array}{ll}
\lambda^{0}=1, & \psi^{0}(T)=\left(I_{*}-I_{0}(T)\right) A_{0}^{-1}(T) \quad \forall T \in \mathbb{T} ; \\
\lambda^{0}=0, \quad \psi^{0}(T)=\quad \iota_{*} A_{0}^{-1}(T) \quad \forall T \in \mathbb{T} .
\end{array}
$$

Core relations of the Maximum Principle are incomplete, since (4a)-(5a) do not contain a condition on the right endpoint, or, which is actually equivalent, on $I_{*}$ or $\iota_{*}$. The remaining part of the paper is mainly devoted to finding the additional relations at $I_{*}$ and $\iota_{*}$ with the aid of $\tau$-vanishing Lagrange multiplier.

\subsection{Existence of $\tau$-Vanishing Multipliers}

System (4a)-(4b) can be rewritten for $u=u^{0}$ in the form

$$
\begin{array}{r}
\dot{\psi}(t)=-\frac{\partial \mathcal{H}}{\partial x}\left(x(t), t, u^{0}(t), \lambda, \psi(t)\right), \\
\dot{x}(t)=f\left(t, x(t), u^{0}(t)\right), \\
\dot{\lambda}=0 .
\end{array}
$$

Definition 2 A nontrivial Lagrange multiplier $\left(\lambda^{0}, \psi^{0}\right)$ associated with $\left(x^{0}, u^{0}\right)$ is called $\tau$-vanishing (or just vanishing) if $\left(\psi^{0}, x^{0}, \lambda^{0}\right)$ is a pointwise limit of a sequence of solutions $\left(\psi_{n}, x_{n}, \lambda_{n}\right)_{n \in \mathbb{N}}$ of system (8a)-(8c) such that $\psi_{n}\left(\tau_{n}^{\prime}\right)=0$ for every $n \in \mathbb{N}$, here $\tau^{\prime} \subset \tau$. In this case, the shadow price $\psi^{0}$ is called $\tau$-vanishing as well.

Geometrically, this property means that the tube of solutions of system (8a)-(8c), however thin (at the initial time), intersects with the hyperplane $\psi=0_{\mathbb{X}}$ at arbitrarily far time $\tau_{n}$.

We claim that the existence of $\tau$-vanishing multipliers is a necessary optimality condition. The main 'work horse' of this proof is the following asymptotic condition of optimality structurally similar to [3, Theorem 9.1], [5, Theorem 3].

Proposition 3 Under conditions $(\mathbf{u}),(\mathbf{f g}),(\tau)$, for each weight $w \in(N u l l)\left(u^{0}\right)$, there exist a sequence $\left(x^{n}, \eta^{n}, \lambda^{n}, \psi^{n}\right)_{n \in \mathbb{N}} \in \widetilde{\mathfrak{Y}}^{\mathbb{N}}$ and a subsequence $\tau^{\prime}$ of $\tau$ such that

(1) for some $\left(x^{0}, \widetilde{u}^{0}, \lambda^{0}, \psi^{0}\right) \in \mathfrak{Z}$ it is $\left(x^{n}, \eta^{n}, \lambda^{n}, \psi^{n}\right) \rightarrow\left(x^{0}, \widetilde{u}^{0}, \lambda^{0}, \psi^{0}\right)$ in the topology of $C_{\text {loc }}(\mathbb{T}, \mathbb{X}) \times \widetilde{\mathfrak{U}} \times[0,1] \times C_{\text {loc }}(\mathbb{T}, \mathbb{X})$; 
(2) $\quad\left\|\mathfrak{L}_{w}\left(\eta^{n}\right)\right\|_{C} \rightarrow 0$;

(3) $\widetilde{J}^{\eta^{n}}\left(\tau_{n}^{\prime}\right)-J^{u^{0}}\left(\tau_{n}^{\prime}\right) \rightarrow 0+; \psi^{n}\left(\tau_{n}^{\prime}\right)=0$ for all $n \in \mathbb{N}$.

The proof of this proposition was repositioned to Appendix.

Note that from $\psi^{n}(0)=-\psi^{n}\left(\tau_{n}^{\prime}\right) A^{\eta^{n}}\left(\tau_{n}^{\prime}\right)+\psi^{n}(0) A^{\eta^{n}}(0) \stackrel{(6 \mathrm{~d})}{=} \lambda^{n} I^{\eta^{n}}\left(\tau_{n}^{\prime}\right)$, we have $\lambda^{n} I^{\eta^{n}}\left(\tau_{n}^{\prime}\right) \rightarrow \psi^{0}(0)$.

Let $E=\mathbb{X} \times \mathbb{X} \times \mathbb{T}, \Xi \triangleq 2 \mathbb{D} \times\left(x_{* *}+2 \mathbb{D}\right) \times[0,1]$. To system (4b), (4a), (8c), let us assign the weight $w$ by means of Proposition 2. Substituting this weight into Proposition 3, we obtain

Remark 1 Under conditions $(\mathbf{u}),(\mathbf{f g}),(\tau)$ there exist a sequence $\left(x^{n}, \eta^{n}, \lambda^{n}, \psi^{n}\right)_{n \in \mathbb{N}} \in$ $\widetilde{\mathfrak{Y}}^{\mathbb{N}}$ and a subsequence $\tau^{\prime}$ of $\tau$ such that

(1) for some $\left(x^{0}, \widetilde{u}^{0}, \lambda^{0}, \psi^{0}\right) \in \mathfrak{Z}$, it is $\left(x^{n}, \eta^{n}, \lambda^{n}, \psi^{n}\right) \rightarrow\left(x^{0}, \widetilde{u}^{0}, \lambda^{0}, \psi^{0}\right)$ in the topology of $C_{l o c}(\mathbb{T}, \mathbb{X}) \times \widetilde{U} \times[0,1] \times C_{l o c}(\mathbb{T}, \mathbb{X})$;

(2) the graphs of functions $\left(\psi^{n}, x^{n}, \lambda^{n}\right)$ are contained within the thinning funnels of solutions of system (8a)-(8c); i.e., for some sequence $\left(\delta_{n}\right)_{n \in \mathbb{N}} \in \mathbb{T}^{\mathbb{N}}, \delta_{n} \downarrow 0$, we have

$$
\varkappa\left(t,\left(\psi^{n}, x^{n}, \lambda^{n}\right)(t)\right) \in\left(\psi^{0}(0), x_{* *}, \lambda^{0}\right)+\delta_{n} \mathbb{D} \times \delta_{n} \mathbb{D} \times\left[-\delta_{n}, \delta_{n}\right] \quad \forall t \in \mathbb{T}, n \in \mathbb{N} ;
$$

(3) $\widetilde{J}^{\eta^{n}}\left(\tau_{n}^{\prime}\right)-J^{u^{0}}\left(\tau_{n}^{\prime}\right) \rightarrow 0+$;

(4) $\lambda^{n} I^{\eta^{n}}\left(\tau_{n}^{\prime}\right) \rightarrow \psi^{0}(0) ; \psi^{n}\left(\tau_{n}^{\prime}\right)=0$ for all $n \in \mathbb{N}$.

Note that $\left(\lambda^{0}, \psi^{0}\right)$ is nontrivial because it satisfies boundary condition (5a) as well as the multipliers $\left(\lambda^{n}, \psi^{n}\right)$. For every $n \in \mathbb{N}$, consider a solution $\left(\psi_{n}, x_{n}, \lambda^{n}\right)$ of (8a)(8c) with the initial conditions $\left(\psi_{n}(0), x_{n}(0), \lambda_{n}\right) \triangleq \varkappa\left(\tau_{n}^{\prime},\left(\psi^{n}\left(\tau_{n}^{\prime}\right), x^{n}\left(\tau_{n}^{\prime}\right), \lambda^{n}\right)\right)$. Then $\psi_{n}\left(\tau_{n}^{\prime}\right)=0_{\mathbb{X}}$. Since $\left(\psi_{n}(0), x_{n}(0), \lambda_{n}\right) \in\left(\psi^{0}(0), x^{0}(0), \lambda^{0}\right)+\delta_{n} \mathbb{D} \times \delta_{n} \mathbb{D} \times\left[-\delta_{n}, \delta_{n}\right]$, we have $\left(\psi_{n}(0), x_{n}(0), \lambda_{n}\right) \rightarrow\left(\psi^{0}(0), x^{0}(0), \lambda^{0}\right)$. Consequently, because of the continuous dependency of solutions of $(8 \mathrm{a})-(8 \mathrm{c}),\left(\lambda^{0}, \psi^{0}\right)$ is a $\tau$-vanishing Lagrange multiplier.

Theorem 2 Assume that conditions (u), (fg), $(\tau)$ hold.

Then, there exists a $\tau$-vanishing Lagrange multiplier $\left(\lambda^{0}, \psi^{0}\right) \in \Lambda$, for example, constructed with a limit of sequences from Remark 1.

Moreover, for every $\tau$-vanishing Lagrange multiplier $\left(\lambda^{0}, \psi^{0}\right) \in \Lambda$, there exist a subsequence $\tau^{\prime}$ of $\tau$, a converging to $0_{\mathbb{X}}$ sequence $\left(\xi^{n}\right)_{n \in \mathbb{N}} \in \mathbb{X}^{\mathbb{N}}$, a converging to $\lambda^{0}$ sequence $\left(\lambda^{n}\right)_{n \in \mathbb{N}} \in(0,1]^{\mathbb{N}}$ such that

$$
\begin{aligned}
\psi^{0}(0) & =\lim _{n \rightarrow \infty} \lambda^{n} I_{\xi^{n}}\left(\tau_{n}^{\prime}\right) \\
\psi^{0}(T) & =\lim _{n \rightarrow \infty} \lambda^{n}\left(I_{\xi^{n}}\left(\tau_{n}^{\prime}\right)-I_{\xi^{n}}(T)\right) A_{\xi^{n}}^{-1}(T) \\
& =\lim _{n \rightarrow \infty} \lambda^{n} \int_{T}^{\tau_{n}^{\prime}} \frac{\partial g}{\partial x}\left(t, x_{\xi^{n}}(t), u^{0}(t)\right) A_{\xi^{n}}(t) d t A_{0}^{-1}(T) .
\end{aligned}
$$

and all the limits are uniform on every compact. 
If, in addition to that, $\lambda^{0}>0$, then we can assume $\lambda_{n}=\lambda^{0}=1$.

Proof The existence of a $\tau$-vanishing Lagrange multiplier $\left(\lambda^{0}, \psi^{0}\right)$ is shown above. By multiplying this nontrivial $\left(\lambda^{0}, \psi^{0}\right)$ by a certain scalar, we can always provide condition (5a); thus, $\left(\lambda^{0}, \psi^{0}\right) \in \Lambda$.

Let $\left(\lambda^{0}, \psi^{0}\right)$ be a $\tau$-vanishing Lagrange multiplier. The sequences $\tau^{\prime},\left(\lambda_{n}\right)_{n \in \mathbb{N}},\left(\psi_{n}\right)_{n \in \mathbb{N}}$ exist by the definition of a $\tau$-vanishing Lagrange multiplier if we define $\xi^{n} \triangleq x_{n}(0)-x^{0}(0)$ for every $n \in \mathbb{N}$. For all $n \in \mathbb{N}$, we have $\psi_{n}\left(\tau_{n}^{\prime}\right)=0_{\mathbb{X}}$. Then, the Cauchy formula (6d) implies $\psi_{n}(T)=\lambda^{n}\left(I_{\xi^{n}}\left(\tau_{n}^{\prime}\right)-I_{\xi^{n}}(T)\right) A_{\xi^{n}}^{-1}(T)$ for every $T \in \mathbb{T}$; thus, $\psi_{n}(0)=\lambda^{n}\left(I_{\xi^{n}}\left(\tau_{n}^{\prime}\right)-I_{\xi^{n}}(T)\right)=\lambda^{n} I_{\xi^{n}}\left(\tau_{n}^{\prime}\right)$. Now, uniformity of the limit $\psi^{0}$ of $\psi_{n}$ yields (9a). Substituting this into (6d), we obtain (9c) for every $T \in \mathbb{T}$. What remains follows from the theorem of continuous dependence of solutions on initial conditions, applied to (8a)-(8c) and (6b).

\subsection{On Different Topologies for the Set of Generalized Controls}

Consider a weight $w^{0} \in(N u l l)\left(u^{0}\right)$. Define $w^{1}$ by the rule $w^{1}(t, u) \triangleq w^{0}(t, u)+\| u-$ $u^{0}(t) \|$ for every $(t, u) \in \mathbb{T} \times \mathbb{U}$. Then, for a subsequence $\left(u_{n}\right)_{n \in \mathbb{N}} \in \mathfrak{U}^{\mathbb{N}}$, from $\| \mathfrak{L}_{w^{1}}[\widetilde{\delta} \circ$ $\left.u_{n}\right] \|_{C} \rightarrow 0$ it follows that $\left\|u_{n}-u^{0}\right\|_{\mathcal{L}^{1}(\mathbb{T}, \mathbb{U})} \rightarrow 0$ (certainly, this does not imply that $\left.u^{0} \in \mathcal{L}^{1}(\mathbb{T}, \mathbb{U})\right)$. Similarly, for any $p \in(0, \infty), v \in B_{\text {loc }}\left(\mathbb{T}, \mathbb{R}_{>0}\right)$, replacing $\left\|u-u^{0}(t)\right\|$ with $v(t)\left\|u-u^{0}(t)\right\|^{p}$ guarantees the convergence of $u_{n}-u^{0} \rightarrow 0$ in the topology of $\mathcal{L}_{v}^{p}(\mathbb{T}, \mathbb{U})$. For every interval $\mathfrak{T} \subset \mathbb{T}$, this extended metric also induces the extended distance $\varrho\left(\eta, u^{0} ; \widetilde{\mathcal{L}}_{v}^{p}(\mathfrak{T}, \mathbb{U})\right)$ on $\widetilde{\mathfrak{U}}$ by the rule

$$
\varrho\left(\eta, u^{0} ; \widetilde{\mathcal{L}}_{\nu}^{p}(\mathfrak{T}, \mathbb{U})\right) \triangleq\left(\int_{\mathfrak{T}} v(t) \int_{U(t)}\left\|u-u^{0}(t)\right\|^{p} d \eta(t) d t\right)^{1 / p} \forall \eta \in \widetilde{\mathfrak{U}} .
$$

Addition of the summand $v(t) R^{p}(t, u)$ (see (29)) provides the uniform convergence $\left\|\dot{y}(t)-a\left(t, y(t), u^{0}(t)\right)\right\|_{\mathcal{L}_{v}^{p}(\mathbb{T}, \mathbb{X})} \rightarrow 0$ by all $\eta \in \mathfrak{U}, y \in \mathfrak{A}[\eta]$ such that $y(0) \in \Xi$.

Let us replace the weight $w$ from Proposition 3 and Remark 1 by a stronger one if necessary. Then, a $\tau$-vanishing Lagrange multiplier $\left(\lambda^{0}, \psi^{0}\right)$ exists as the limit of sequences from Remark 1.

Remark 2 Assume that conditions $(\mathbf{u}),(\mathbf{f g}),(\tau)$ hold. Then there exists a $\tau$-vanishing multiplier $\left(\lambda^{0}, \psi^{0}\right)$ associated with $\left(x^{0}, u^{0}\right)$ such that for this multiplier, the conclusion of Remark 1 holds and, moreover, the following convergences are guaranteed: $\varrho\left(\eta^{n}, u^{0} ; \widetilde{\mathcal{L}}_{v}^{p}(\mathbb{T}, \mathbb{U})\right) \rightarrow 0,\left\|\dot{x}^{n}(t)-f\left(t, x^{n}(t), u^{0}(t)\right)\right\|_{\mathcal{L}_{v}^{p}(\mathbb{T}, \mathbb{X})} \rightarrow 0_{\mathbb{X}}$.

The condition (u) implies that, a.a. $t \in \mathbb{T}$, the controls are chosen from the compact $U(t)$. Let us weaken this assumption to the following:

Condition $\left(\boldsymbol{u}_{\sigma}\right): \quad U$ is a closed-valued map, and its graph is Borel set.

We still assume the conditions $(\mathbf{f g}),(\tau)$ to hold. A nondecreasing sequence $\left(U^{(r)}\right)_{r \in \mathbb{N}}$ of locally bounded compact-valued maps is given by

$$
U^{(r)}(t) \triangleq\left\{u \in U(t) \mid\left\|u-u^{0}(t)\right\|<r\right\} \quad \forall t \in \mathbb{N}, r \in \mathbb{N} .
$$


Let the set $\mathfrak{U}^{(r)}$ be the set of all Borel measurable selectors of the multi-valued map $U^{(r)}$. Then for all $r \in \mathbb{N} u^{0} \in \mathfrak{U}^{(r)} \subset \mathfrak{U}^{(r+1)}$ and $U \equiv \cup_{r \in \mathbb{N}} U^{(r)}$ hold; now, we have $\mathfrak{U}^{(\infty)} \triangleq \cup_{r \in \mathbb{N}} \mathfrak{U}^{(r)} \equiv \mathfrak{U}$.

Repeating the reasonings of Section 3, for every $r \in \mathbb{N} \cup\{\infty\}$, we can construct sets $\widetilde{\mathfrak{U}}^{(r)}$ and images $\mathfrak{U}_{n}^{(r)} \triangleq \pi_{n}\left(\mathfrak{U}^{(r)}\right), \widetilde{\mathfrak{U}}_{n}^{(r)} \triangleq \widetilde{\pi}_{n}\left(\widetilde{\mathfrak{U}}^{(r)}\right)$. Denote by $\widetilde{\mathfrak{U}}$ the set of all maps $\eta$ from $\mathbb{T}$ into the set of Radon probability measures over $\mathbb{U}$ such that $\left.\eta\right|_{[0, n]} \in \widetilde{\mathfrak{U}}_{n}^{(\infty)}$ for every $n \in \mathbb{N}$. The topology of this set is the weakest topology in which $\mathfrak{U}^{(r)}$ could be continuously embedded into $\widetilde{\mathfrak{U}}$. Note that under our definition, $\widetilde{u}^{0} \in \widetilde{\delta}\left(\mathfrak{U}^{(r)}\right) \subset \widetilde{\delta}(\mathfrak{U})$ for all $r \in \mathbb{N}$.

To system (4b), (4a), and (8c), let us assign the weight $w$ by means of Proposition 2. Note that this weight depends only on $\Xi, f, g$, and $u^{0}$, and is independent of the multi-valued map $U$ (see (29)). For the sequence $\tau$, for each $\widetilde{\mathfrak{U}}^{(r)}$, we have Remark 1; in particular, there exist a time $t_{r} \in \tau\left(t_{r}>r\right)$, a $\tau$-vanishing Lagrange multiplier $\left(\lambda^{r}, \psi^{r}\right)$, and a solution $\left(x^{r}, \eta^{r}, \widetilde{\psi}^{r}, \widetilde{\lambda}^{r}\right) \in \widetilde{\mathfrak{Y}}$ with the properties

$$
\begin{array}{r}
\sup _{p \in U^{(r)}(t)} \mathcal{H}\left(x(t), t, p, \lambda^{r}, \psi^{r}(t)\right)=\mathcal{H}\left(x(t), t, u^{0}(t), \lambda^{r}, \psi^{r}(t)\right) \forall \text { a.a.t } \in \mathbb{T} \\
\left\|\mathfrak{L}_{w}\left(\eta^{r}\right)\right\|_{C}<1 / r,\left\|\widetilde{x}^{r}-x^{r}\right\|_{C([0, r], \mathbb{X})}<1 / r,\left\|\widetilde{\psi}^{r}-\psi^{r}\right\|_{C([0, r], \mathbb{X})}<1 / r \\
\left\|\varkappa\left(t_{r},\left(\widetilde{\psi}^{r}\left(t_{r}\right), \widetilde{x}^{r}\left(t_{r}\right), \tilde{\lambda}^{r}\right)\right)-\left(\psi^{0}, x_{* *}, \lambda^{0}\right)\right\|_{E}<1 / r \\
0 \leq \widetilde{J}^{\eta^{r}}\left(t_{r}\right)-J^{u^{0}}\left(t_{r}\right)<1 / r, \widetilde{\psi}^{r}\left(t_{r}\right)=0_{\mathbb{X}} .
\end{array}
$$

Passing to the limit, we obtain $\eta^{r} \rightarrow \widetilde{u^{0}}$ from $\left\|\mathfrak{L}_{w}\left(\eta^{r}\right)\right\|_{C}<1 / r$. Passing to the subsequence $\tau^{\prime} \subset\left(t_{r}\right)_{r \in \mathbb{N}} \subset \tau$, we can provide the monotonicity of $t_{r}$ and convergence of the sequence of $\left(\lambda^{r}, \psi^{r}\right) \in(0,1] \times C_{l o c}(\mathbb{T}, \mathbb{X})$ to certain $\left(\lambda^{0}, \psi^{0}\right)$. Under these assumptions, we immediately see that $\left(\psi^{0}, x^{0}, \lambda^{0}\right)$ is the solution of $(8 \mathrm{a})-(8 \mathrm{c})$ that satisfies (5a). Now, the sequence of $\left(\widetilde{x}^{r}, \eta^{r}, \widetilde{\psi}^{r}, \tilde{\lambda}^{r}\right)$ converges, by (10b), to $\left(x^{0}, \widetilde{u}^{0}, \lambda^{0}, \psi^{0}\right)$. Passing to the pointwise limit in (10a), we obtain the property (4c) for $\left(x^{0}, \widetilde{u}^{0}, \lambda^{0}, \psi^{0}\right)$. Thus we proved items (1) and (2) of Remark 1. Since the limit of $\left(\tilde{x}^{r}, \eta^{r}, \widetilde{\psi}^{r}, \tilde{\lambda}^{r}\right)_{r \in \mathbb{N}}$ and $\left(x^{0}, \eta^{r}, \psi^{r}, \lambda^{r}\right)_{r \in \mathbb{N}}$ is the same, items (3) and (4) follow from (10c) and (10d), respectively.

Consider again the solutions $\left(\psi_{n}, x_{n}, \lambda^{n}\right)$ of $(8 \mathrm{a})-(8 \mathrm{c})$ for the initial conditions $\left(\psi_{n}(0), x_{n}(0), \lambda_{n}\right) \triangleq \varkappa\left(\tau_{n}^{\prime},\left(\widetilde{\psi}^{n}\left(\tau_{n}^{\prime}\right), \widetilde{x}^{n}\left(\tau_{n}^{\prime}\right), \tilde{\lambda}^{n}\right)\right)$. Then, $\left(\lambda^{0}, \psi^{0}\right)$ is a $\tau$-vanishing Lagrange multiplier and Theorem 2 holds under condition $\left(\mathbf{u}_{\sigma}\right)$. Thus,

Corollary 1 Condition (u) in Remark 1, Theorem 2 could be replaced with $\left(\mathbf{u}_{\sigma}\right)$.

Corollary 2 Assume conditions $\left(\mathbf{u}_{\sigma}\right)$, (fg) hold. Let a pair $\left(x^{0}, u^{0}\right) \in C_{\text {loc }}(\mathbb{T}, \mathbb{X}) \times \mathfrak{U}$ be a weakly uniformly overtaking optimal for problem (1a)-(1b).

Then, for some unbounded sequence $\tau=\left(\tau_{n}\right)_{n \in \mathbb{N}} \in \mathbb{T}^{\mathbb{N}}$, there exists a $\tau$-vanishing Lagrange multiplier $\left(\lambda^{0}, \psi^{0}\right) \in \Lambda$.

Corollary 3 Assume conditions $\left(\mathbf{u}_{\sigma}\right)$, (fg) hold. Let a pair $\left(x^{0}, u^{0}\right) \in C_{\text {loc }}(\mathbb{T}, \mathbb{X}) \times \mathfrak{U}$ be a uniformly overtaking optimal for problem (1a)-(1b).

Then, for each unbounded sequence $\tau=\left(\tau_{n}\right)_{n \in \mathbb{N}} \in \mathbb{T}^{\mathbb{N}}$, there exists a $\tau$-vanishing Lagrange multiplier $\left(\lambda^{0}, \psi^{0}\right) \in \Lambda$. 


\section{Properties of $\tau$-Vanishing Lagrange Multipliers}

\subsection{On Stable Shadow Prices}

Consider the boundary conditions

$$
\begin{array}{r}
\lim _{t \rightarrow \infty} \psi(t)=0, \\
\liminf _{n \rightarrow \infty}\left\|\psi^{0}\left(\tau_{n}\right)\right\|_{\mathbb{X}}=0 .
\end{array}
$$

Definition 3 The component $\psi^{0}$ of a solution $y^{0}=\left(\psi^{0}, x^{0}, \lambda^{0}\right)$ of system (8a)-(8c) is said to be Lyapunov stable in domain $\Xi$ if for each $\varepsilon>0$ there exists $\delta>0$ such that for each solution $y=(\psi, x, \lambda)$ of system $(8 \mathrm{a})-(8 \mathrm{c})$ from $\left\|y(0)-y^{0}(0)\right\|_{E}<\delta, y(0) \in$ $\Xi$ it follows that $\left\|\psi^{0}(s)-\psi(s)\right\|_{\mathbb{X}}<\varepsilon$ for all $s \in \mathbb{T}$.

Corollary 4 Assume that conditions $\left(\mathbf{u}_{\sigma}\right),(\mathbf{f g}),(\tau)$ hold. Let for some solution $\left(\psi, x^{0}, \lambda\right)$ of system $(8 \mathrm{a})-(8 \mathrm{c})$ the component $\psi$ be Lyapunov stable in the domain $\mathbb{X} \times \mathbb{X} \times[0,1]$

Then all $\tau$-vanishing multipliers $\left(\lambda^{0}, \psi^{0}\right) \in \Lambda$ satisfy the condition (11b).

Proof Since equation (8a) is linear, the Lyapunov stability of $\psi$ for some solution $\left(\psi, x^{0}, \lambda\right)$ of system $(8 \mathrm{a})-(8 \mathrm{c})$ yields the Lyapunov stability of this component for all solutions of system $(8 \mathrm{a})-(8 \mathrm{c})$.

Consider every $\tau$-vanishing multiplier $\left(\lambda^{0}, \psi^{0}\right)$ and the sequences $\tau^{\prime},\left(\lambda_{n}\right)_{n \in \mathbb{N}}$, $\left(x_{n}\right)_{n \in \mathbb{N}},\left(\psi_{n}\right)_{n \in \mathbb{N}}$ from its definition. Then, $y_{n}=\left(\psi_{n}(0), x_{n}(0), \lambda_{n}\right) \rightarrow y^{0}=$ $\left(\psi^{0}(0), x^{0}(0), \lambda^{0}\right)$; and, for some $N \in \mathbb{N}$ for all $n \in \mathbb{N}, n>N$, from the definition of Lyapunov stability it follows that $\left\|\psi^{0}\left(\tau_{n}^{\prime}\right)\right\|_{\mathbb{X}}=\left\|\psi^{n}\left(\tau_{n}^{\prime}\right)-\psi^{0}\left(\tau_{n}^{\prime}\right)\right\|_{\mathbb{X}}<\varepsilon$. Since $\varepsilon>0$ was arbitrary, we have shown (11b) for all $\tau$-vanishing multipliers.

Note that since (4b) is linear, the partial stability of the variable $\psi$ implies its boundedness. Therefore, the proved proposition is useless if all shadow prices are unbounded. Note that, for every weakly uniformly overtaking optimal control $u^{0}$, a solution $\left(x^{0}, u^{0}, \lambda^{0}, \psi^{0}\right) \in \mathfrak{Z}$ that satisfies (11b) may not satisfy stronger condition (11a) (see [41, Example 5.1], Example 2).

The stability condition can be replaced with a condition which is stronger but much easier to check.

Corollary 5 Assume that conditions $\left(\mathbf{u}_{\sigma}\right),(\mathbf{f g}),(\tau)$ hold. If the functions $L_{K}^{f}, L_{K}^{g}$ are independent of the compact $K$, and these functions are summable on $\mathbb{T}$ (see [35, Hypotesis 3.1 (iv)]), then any $\tau$-vanishing multiplier satisfies condition (11a).

Proof Let $\left(\psi^{0}, \lambda^{0}\right)$ be a $\tau$-vanishing multiplier. Let $\xi_{0} \triangleq\left(\psi^{0}(0), x^{0}(0), \lambda^{0}\right), \Xi \triangleq \xi_{0}+$ $\mathbb{D} \times \mathbb{D} \times[-1,1]$. By $[35,(3.3)]$ there exists a summable function $\omega \in \mathfrak{L}^{1}(\mathbb{T}, \mathbb{T})$ such that $\dot{\psi}(t) \leq \omega(t)$ for a.a. $t \in \mathbb{T}$ for all solution $(\psi, x, \lambda)$ of system $(8 \mathrm{a})-(8 \mathrm{c})$ if $\xi \triangleq$ $(\psi(0), x(0), \lambda) \in \Xi$. Now for each pair $\left(t_{1}, t_{2}\right) \in \mathbb{T}, t_{1} \leq t_{2}$,

$$
\left\|\psi\left(t_{1}\right)-\psi^{0}\left(t_{2}\right)\right\|_{\mathbb{X}} \leq\left\|\psi-\psi^{0}\right\|_{C\left(\left[0, t_{1}\right], \mathbb{X}\right)}+2 \int_{t_{1}}^{\infty} \omega(t) d t
$$


if $\xi \in \Xi$. For each $\varepsilon>0$ there exists $T \in \mathbb{T}$ such that the second summand does not exceed $\varepsilon / 2$ if $t_{1}>T$; now there exists $r \in \mathbb{T}$ such that $\left\|\psi-\psi^{0}\right\|_{C([0, T], \mathbb{X})}$ does not exceed $\varepsilon / 2$ if $\left\|\xi-\xi^{0}\right\|_{E}<r$. Then, setting $t_{1}=t_{2}$, we obtain $\left\|\psi-\psi^{0}\right\|_{C} \leq \varepsilon$ if $\| \xi_{1}-$ $\xi_{2} \|_{E}<r$, i.e., the component $\psi^{0}$ is Lyapunov stable. By Corollary 4 , condition (11b) holds, and $\left\|\psi^{0}\left(T_{1}\right)\right\|<\varepsilon / 2$ for some $T_{1}>T$.

Then, setting $\xi=\left(\psi^{0}(0), x^{0}(0), \lambda^{0}\right)$, we obtain $\left\|\psi^{0}\left(t_{2}\right)\right\|_{\mathbb{X}}=\left\|\psi^{0}\left(t_{2}\right)-\psi^{0}\left(T_{1}\right)\right\|_{\mathbb{X}}+$ $\left\|\psi^{0}\left(T_{1}\right)\right\|_{\mathbb{X}}<\varepsilon$ if $t_{2}>T_{1}$. Thus (11a) holds.

The even more strong conditions used for proving transversality condition (11a) can be seen, for example, in [48, (A3)] (the Lipshitz constants $L_{K}^{g}, L_{K}^{f}$ are required to decrease exponentially with time).

\subsection{Degenerate $\tau$-Vanishing Lagrange Multipliers}

Remark 3 Assume that conditions $\left(\mathbf{u}_{\sigma}\right),(\mathbf{f g}),(\tau)$ hold. If for some $\tau^{\prime} \subset \tau$

$$
\limsup _{n \rightarrow \infty, \xi \rightarrow 0_{\mathbb{X}}}\left\|I_{\xi}\left(\tau_{n}^{\prime}\right)\right\|_{\mathbb{X}}<\infty
$$

then the pair $\left(x^{0}, u^{0}\right)$ is normal, and there exists a $\tau$-vanishing multiplier $\left(1, \psi^{0}\right) \in \Lambda$.]

Moreover, if $\limsup \left\|I_{\xi}\left(\tau_{n}\right)\right\|_{\mathbb{X}}<\infty$, then every $\tau$-vanishing multiplier $\left(\lambda^{0}, \psi^{0}\right) \in \Lambda$ satisfies $\lambda^{0}=1$.

On the other hand, if $\lim _{n \rightarrow \infty, \xi \rightarrow 0 \mathbb{X}}\left\|I_{\xi}\left(\tau_{n}\right)\right\|_{\mathbb{X}}=\infty$, then every $\tau$-vanishing multiplier $\left(\lambda^{0}, \psi^{0}\right) \in \Lambda$ satisfies $\lambda^{0}=0$.

Proof By Theorem 2, there exists a $\tau$-vanishing multiplier $\left(\lambda^{0}, \psi^{0}\right) \in \Lambda$ satisfying (9a), but for each such multiplier, we have $\lambda^{n}|| I_{\xi_{n}}\left(\tau_{n}^{\prime}\right)\left\|_{\mathbb{X}}=\right\| \psi^{n}(0)\left\|_{\mathbb{X}} \rightarrow\right\| \psi^{0}(0) \|_{\mathbb{X}}$; then $\lambda^{0}=0$ iff $\left(\left\|I_{\xi_{n}}\left(\tau_{n}^{\prime}\right)\right\|_{\mathbb{X}}\right)_{n \in \mathbb{N}} \uparrow \infty$.

Note that if $\limsup _{n \rightarrow \infty}\left\|I_{0}\left(\tau_{n}\right)\right\|_{E}<\infty$, then $\tau$-vanishing multiplier $\left(\lambda^{0}, \psi^{0}\right) \in \Lambda$ can satisfy $\lambda^{0}=0$ (see Example 4$)$.

There are many conditions that provide nondegeneracy of the problem; see [3, $5,7,9,36]$. The connection between the normality of the problem and finiteness of $I_{0}$ seems to be noted for the first time in [3, (3.24)]. Condition (12) develops this approach, actually demanding $I_{\xi}$ to be locally bounded. As we are going to show below, many sufficient conditions of nondegeneracy for the optimal problem can be obtained from (12). However, there are other ways to prove the nondegeneracy. For example, [3, Theorem 5.1] uses the smoothness of the objective value function, and [3, Theorem 10.1] and [5, Theorem 5] use the monotonicity of the functions $f$ and $g$ in $x$ and the stationarity condition.

Note that although the examples of abnormal problems are well known [3, 23, 33], additional relations of the Maximum Principle for such problems did not receive much attention from researchers; the author only knows of the dual problem construction in paper [33]. Let us apply Theorem 2 to these problems. 
Consider a degenerate $\tau$-vanishing solution $\left(x^{0}, u^{0}, 0, \psi^{0}\right) \in \mathfrak{Z}$. Then, from (5a) we have $\psi^{0}(0)=1$, and Theorem 2 yields

$$
\psi^{0}(0) \stackrel{(9 \mathrm{a})}{=} \frac{\psi^{0}(0)}{\left\|\psi^{0}(0)\right\|_{\mathbb{X}}}=\lim _{n \rightarrow \infty} \frac{\lambda_{n} I_{x_{n}(0)}\left(\tau_{n}^{\prime}\right)}{\left\|\lambda_{n} I_{x_{n}(0)}\left(\tau_{n}^{\prime}\right)\right\|_{\mathbb{X}}}=\lim _{n \rightarrow \infty} \frac{I_{x_{n}(0)}\left(\tau_{n}^{\prime}\right)}{\left\|I_{x_{n}(0)}\left(\tau_{n}^{\prime}\right)\right\|_{\mathbb{X}}}
$$

provided $x_{n}(0) \rightarrow x^{0}(0)$. Using Remark 3 , we finally obtain

Corollary 6 Let $\left(\mathbf{u}_{\sigma}\right),(\mathbf{f g}),(\tau)$ hold. Let

$$
\lim _{n \rightarrow \infty, \xi \rightarrow 0_{\mathbb{X}}}\left\|I_{\xi}\left(\tau_{n}\right)\right\|_{\mathbb{X}}=\infty, \quad \lim _{n \rightarrow \infty, \xi \rightarrow 0_{\mathbb{X}}} \frac{I_{\xi}\left(\tau_{n}\right)}{\left\|I_{\xi}\left(\tau_{n}\right)\right\|_{\mathbb{X}}}=\iota_{*} .
$$

for some vector $\iota_{*} \in \mathbb{X}$.

Then, there exists a unique $\tau$-vanishing multiplier $\left(0, \psi^{0}\right) \in \Lambda$, and $\iota_{*}$ and $\psi^{0}$ are connected by (7b).

\subsection{Monotonic Case}

Consider a nonempty convex closed cone $\mathfrak{C} \subset \mathbb{X}$, and its interior int $\mathfrak{C}$. The cone orderings $\succcurlyeq, \succ$ of $\mathbb{X}$ induced by $\mathfrak{C}$ are the relations defined as follows: for all $x, y \in \mathbb{X}$,

$$
\left(x \succcurlyeq_{\mathfrak{C}} y\right) \Leftrightarrow(x-y \in \mathfrak{C}), \quad\left(x \succ_{\mathfrak{C}} y\right) \Leftrightarrow(x-y \in \text { int } \mathfrak{C}) .
$$

The pre-orders on $\mathbb{L}$ are defined as follows: for $B, C \in \mathbb{L}$,

$$
\begin{aligned}
& \left(B \succcurlyeq_{\mathfrak{C}} C\right) \Leftrightarrow((B-C) x \in \mathfrak{C} \quad \forall x \in \mathfrak{C}), \\
& \left.\left(B \succ_{\mathfrak{C}} C\right) \Leftrightarrow \in \text { int } \mathfrak{C} \quad \forall x \in \text { int } \mathfrak{C}\right) .
\end{aligned}
$$

Note that $1_{\mathbb{L}} \succcurlyeq_{\mathfrak{C}} 0_{\mathbb{L}}, 1_{\mathbb{L}} \succ_{\mathfrak{C}} 0_{\mathbb{L}}$.

The conjugate cone of $\mathfrak{C}$ is defined by $\mathfrak{C}^{\perp} \triangleq\{x \in \mathbb{X} \mid \forall y \in \mathfrak{C} x y \geq 0\}$.

Proposition 4 Assume that conditions $\left(\mathbf{u}_{\sigma}\right),(\mathbf{f g}),(\tau)$ hold. Assume that there exists a Caratéodory function $d: \mathbb{T} \times \mathbb{X} \rightarrow \mathbb{R}$ such that for all $x \in \mathbb{X}$ and a.a. $t \in \mathbb{T}$ the following relation holds:

$$
\frac{\partial g}{\partial x}\left(t, x, u^{0}(t)\right) \succcurlyeq_{\mathfrak{C}^{\perp}} 0_{\mathbb{L}}, \quad \frac{\partial f}{\partial x}\left(t, x, u^{0}(t)\right) \succcurlyeq_{\mathfrak{C}} d(t, x) 1_{\mathbb{L}} .
$$

Then, there exists a $\tau$-vanishing multiplier $\left(\lambda^{0}, \psi^{0}\right) \in \Lambda$, and for every such multiplier, we have $\psi^{0} \succcurlyeq_{\mathfrak{C}^{\perp}} 0_{\mathbb{X}}$, and $\psi^{0}(0) \in \mathfrak{C}^{\perp}$.

Moreover, if $\lambda^{0}>0$ (for example, if (12) holds), then for all $y \in \mathfrak{C}$

$$
\limsup _{t \rightarrow \infty, \xi \rightarrow 0_{\mathbb{X}}} I_{\xi}(t) y \geq \psi^{0}(0) y \geq \lim _{t \rightarrow \infty} I_{0}(t) y \geq 0,
$$

and all limits in (14) are correctly defined.

In addition, if there exists a Lebesgue point $t^{*} \in \mathbb{T}$ for the function $u^{0}$ such that

$$
\frac{\partial g}{\partial x}\left(t^{*}, x^{0}\left(t^{*}\right), u^{0}\left(t^{*}\right)\right) \succ_{\mathfrak{C}^{\perp}} 0_{\mathbb{L}},
$$

then $\left.\psi^{0}\right|_{\left[0, t^{*}\right]} \succ_{\mathfrak{C}^{\perp}} 0_{\mathbb{X}}$; in particular, $\psi^{0}(0)$ is contained the interior of $\mathfrak{C}^{\perp}$. If such Lebesgue point $t^{*}$ exists on every infinite interval, then $\psi^{0} \succ_{\mathfrak{C}^{\perp}} 0$. 
Proof Fix arbitrary $\xi \in \mathbb{X}, T>0, \quad \vartheta>T$. Denote by $F_{\xi}(t)$ the matrix $\frac{\partial f}{\partial x}\left(t, x_{\xi}(t), u^{0}(t)\right)$, and by $m_{\xi}$ the measurable function $t \mapsto-d\left(t, x_{\xi}(t)\right)$; by condition, $F_{\xi}+m_{\xi}(t) 1_{\mathbb{L}} \succcurlyeq_{\mathfrak{C}} 0_{\mathbb{L}}$. Now, let us consider a solution $P(t)$ of the equation

$$
\dot{P}=\left(F_{\xi}(t)+m_{\xi}(t) 1_{\mathbb{L}}\right) P, \quad P(T)=1_{\mathbb{L}}, \quad t \geq T
$$

then $P(t) \succcurlyeq_{\mathfrak{C}} 1_{\mathbb{L}}$ for all $t \in(T, \vartheta]$. But the solution $P$ is the product of two nonnegative solutions of the equations $\dot{Q}=F_{\xi}(t) Q, Q(T)=1_{\mathbb{L}}$, and $\dot{r}_{\xi}=$ $m_{\xi}(t) r_{\xi}, r_{\xi}(T)=1$. Thus, $P(\vartheta)=r_{\xi}(\vartheta) Q(\vartheta)=r_{\xi}(\vartheta) A_{\xi}(\vartheta) A_{\xi}^{-1}(T)$, and $P(\vartheta) \succcurlyeq_{\mathfrak{C}} 1_{\mathbb{L}}$ implies $A_{\xi}(\vartheta) A_{\xi}^{-1}(T)=Q(\vartheta)=P(\vartheta) / r_{\xi}(\vartheta) \succcurlyeq_{\mathfrak{C}} 1_{\mathbb{L}} / r_{\xi}(\vartheta)$ for all $\vartheta>T$. In particular, for all $y \in \mathfrak{C}$, we have $A_{\xi}(\vartheta) A_{\xi}^{-1}(T) y \succcurlyeq_{\mathfrak{C}} y / r_{\xi}(\vartheta)$, whence

$$
\frac{d I_{\xi}(t)}{d t} A_{\xi}^{-1}(T) y=\frac{\partial g}{\partial x}\left(t, x_{\xi}(t), u^{0}(t)\right) A_{\xi}(t) A_{\xi}^{-1}(T) y \geq \frac{\partial g}{\partial x}\left(t, x_{\xi}(t), u^{0}(t)\right) \frac{y}{r_{\xi}(t)} \geq 0
$$

for all $\xi \in \mathbb{X}, y \in \mathfrak{C}, T \in \mathbb{T}, t>T$. In particular, for $T=0$, we obtain $\frac{d I_{\xi}(t)}{d t} \in \mathfrak{C}^{\perp}$. Thus, the functions $I_{\xi} y, I_{\xi} A_{\xi}^{-1}(T) y$ are monotonic in $t$ for all $\xi \in \mathbb{X}, T \in \mathbb{T}, y \in \mathfrak{C}$.

By Theorem 2, there exists a $\tau$-vanishing multiplier $\left(\psi^{0}, \lambda^{0}\right) \in \Lambda$. Moreover, each such multiplier $\left(\psi^{0}, \lambda^{0}\right) \in \Lambda$ satisfies formula (9c) for certain sequences $\lambda^{n}$ and $\xi_{n}$. However, the integrand of (9c) lies in $\mathfrak{C}^{\perp}$. Passing to the limit as $n \rightarrow \infty$, we obtain $\psi^{0} \succcurlyeq \mathfrak{C}^{\perp} 0_{\mathbb{X}}$.

Fix the basis of $\operatorname{span} \mathfrak{C}$ made of the vectors $y \in \mathfrak{C}$; now, for every such vector $y$, the functions $I_{\xi} y$ are monotonic, and

$$
\limsup _{t \rightarrow \infty, \xi \rightarrow 0 \mathbb{X}} \lambda_{0} I_{\xi}(t) y \geq \lim _{n \rightarrow \infty} \lambda_{0} I_{\xi_{n}}\left(\tau_{n}^{\prime}\right) y \stackrel{(9 \mathrm{a})}{=} \psi^{0}(0) y
$$

we obtain the first estimate from (14).

Fix any $T \in \mathbb{T}, y \in \mathfrak{C}$. Now, monotonicity of $I_{\xi} A_{\xi}^{-1}(T) y$ yields

$$
\begin{aligned}
\psi^{0}(T) y & \stackrel{(9 \mathrm{~b})}{=} \lim _{n \rightarrow \infty} \lambda^{n}\left(I_{\xi_{n}}\left(\tau_{n}^{\prime}\right)-I_{\xi_{n}}(T)\right) A_{\xi_{n}}^{-1}(T) y \geq \lim _{n \rightarrow \infty} \lambda^{n}\left(I_{\xi_{n}}(t)-I_{\xi_{n}}(T)\right) A_{\xi_{n}}^{-1}(T) y \\
& =\lambda^{0}\left(I_{0}(t)-I_{0}(T)\right) A_{0}^{-1}(T) y \stackrel{(15)}{\geq} \lambda^{0} \int_{T}^{t} \frac{\partial g}{\partial x}\left(\vartheta, x^{0}(\vartheta), u^{0}(\vartheta)\right) \frac{y d \vartheta}{r_{0}(\vartheta)} \geq 0 \quad \forall t>T .
\end{aligned}
$$

Substituting $T=0$ and passing to the limit as $t \rightarrow \infty$, we obtain the lower estimate from (14).

If $\lambda^{0}>0$, and, in addition, there exists the Lebesgue point $t^{*}$ with the required property, then for all points $T \leq t^{*}, t>t^{*}$, sufficiently close to $t^{*}$, integration on $[T, t]$ yields " $>$ " instead of " $\geq$ " in the latter inequality of (16). Since by (15) this integrand is nonnegative for all $t \in \mathbb{T}$, the same is true for all $T \leq t^{*}, t>t^{*}$, whence we obtain $\left.\psi^{0}\right|_{\left[0, t^{*}\right]} \succ_{\mathfrak{C}} 0_{\mathbb{X}}$.

Regarding the latter point, note that if we have $\psi(t) \nsucc_{\mathfrak{C}} 0_{\mathbb{X}}$ for some $t \in \mathbb{T}$, then taking $t^{*}$ from $(t, \infty)$ yields a contradiction.

Remark 4 For $\psi^{0}(0) \succ_{\mathfrak{C}} 0$, it is sufficient to find for each vector $y_{i}$ from some basis of span $\mathfrak{C}^{\mathfrak{c}}$ Lebesgue point $t_{i}^{*}$ with the property $\frac{\partial g}{\partial x}\left(t_{i}^{*}, x^{0}\left(t_{i}^{*}\right), u^{0}\left(t_{i}^{*}\right)\right) y_{i}>0$. 
Let the right-hand side of the dynamics equation and the integrand of the objective functional be monotonic in $x$. This case frequently arises in economical applications, and monotonicity simplifies its analysis. It seems that the first to note the peculiarities of this case and to investigate it were Aseev, Kryazhimskii, and Taras'ev in their paper [6]. These were followed by papers [1, 45]; the most general cases were considered in $[3,5]$.

Fix the cone $\mathfrak{C} \triangleq \mathbb{T}^{\operatorname{dim} \mathbb{X}}$. In this case, $\mathfrak{C}^{\perp}=\mathfrak{C}$. Replace $\succcurlyeq_{\mathbb{T d i m} \mathbb{X}}, \succ_{\mathbb{T} \text { dim } \mathbb{X}}$ with $\succcurlyeq, \succ$. We obtain

Corollary 7 Assume conditions $\left(\mathbf{u}_{\sigma}\right),(\mathbf{f g}),(\tau)$ hold. Assume that, for all $x \in \mathbb{X}$ and for a.a. $t \in \mathbb{T}$, the matrix $\frac{\partial f}{\partial x}\left(t, x, u^{0}(t)\right)$ is a matrix with nonnegative off-diagonal entries, and $\frac{\partial g}{\partial x}\left(t, x, u^{0}(t)\right)$ is a nonnegative covector, i.e., there exists a number $d(t, x) \in \mathbb{R}$ such that the following relation holds:

$$
\frac{\partial g}{\partial x}\left(t, x, u^{0}(t)\right) \succcurlyeq 0_{\mathbb{X}}, \quad \frac{\partial f}{\partial x}\left(t, x, u^{0}(t)\right) \succcurlyeq d(t, x) 1_{\mathbb{L}} .
$$

Then, there exists a $\tau$-vanishing multiplier $\left(\lambda^{0}, \psi^{0}\right) \in \Lambda$, and for every such multiplier we have $\psi^{0} \succcurlyeq 0_{\mathbb{X}}$, and

$$
\lambda^{0} \limsup _{t \rightarrow \infty, \xi \rightarrow 0_{\mathbb{X}}} I_{\xi}(t) \succcurlyeq \psi^{0}(0) \succcurlyeq \lambda^{0} \lim _{t \rightarrow \infty} I_{0}(t) \succcurlyeq 0_{\mathbb{X}}
$$

holds, and all limits in (18) are correctly defined and finite.

If $\lambda^{0}>0$ (for example, under (12)) and there exists a Lebesgue point $t^{*} \in \mathbb{T}$ for the control $u^{0}$ such that

$$
\frac{\partial g}{\partial x}\left(t^{*}, x^{0}\left(t^{*}\right), u^{0}\left(t^{*}\right)\right) \succ 0_{\mathbb{L}}
$$

we have $\left.\psi^{0}\right|_{\left[0, t^{*}\right]} \succ 0_{\mathbb{X}}$; in particular, $\psi^{0}(0) \succ 0_{\mathbb{X}}$.

Remark 5 Assume that under conditions of Corollary 7, we can choose $d(t, x) \equiv 0$, and the integral

$$
\int_{0}^{t} \frac{\partial g}{\partial x}\left(\vartheta, x^{0}(\vartheta), u^{0}(\vartheta)\right) d \vartheta
$$

unboundedly increases as $t \rightarrow \infty$; then, all $\tau$-vanishing solutions are degenerate.

Indeed, under $d(t, x) \equiv 0$, we can assume $r_{0} \equiv 1$; then, in the case $\lambda^{0}>0,(16)$ will, for $T=0$, imply the boundedness of this integral.

Note that in [6, Theorem 1], [3, Theorem 10.1], and [5, Theorem 5], the estimate $\psi \succcurlyeq 0_{\mathbb{X}}$ is made for problems

$$
\dot{x}=f(x, u), u \in U, x(0)=x_{0}, \quad \int_{0}^{\infty} e^{-\rho t} g(x(t), u(t)) d t \rightarrow \max .
$$

The most general case is examined in [5, Theorem 5]; namely, a variant of Corollary 7 is stated: if (17) is satisfied for all $t \in \mathbb{T}, u \in U(t), x \in \mathbb{X}$ (see [5, (A8)]), then $\psi \succcurlyeq 0_{\mathbb{X}}$, and estimate (18) holds (see [5, (5.5)]); the conditions, under which $\psi \succ 0_{\mathbb{X}}$ holds in addition to the above, are also specified. The explicit form of estimate (18) under the very strong conditions on $f$ and $g$ is also specified in [45, (23)-(26)]. 
Let us also remark that in all papers mentioned, the nondegeneracy of the problem was not assumed (and was not directly reduced to inequality (12)), it had to be proved. For example in [5, Theorem 5], it is demonstrated with the aid of the stationarity condition from additional proposition [5, (A7)]: on any admissible trajectory, there are some $(t, u)$, for which $f(x(t), u) \succ 0_{\mathbb{X}}$.

Note that Example 4 satisfies all assumptions of [5, Theorem 5] and Corollary 7 with the unique relaxation: the function $\frac{\partial g}{\partial x}(t, x, u)$ is nonnegative for all $u \in \mathfrak{U}, t \in$ $\mathbb{T},\left\|x-x^{0}(t)\right\| \leq r$ only for some $r \in(0,1 / 2)$. The nonnegativity of $\psi$, inequality (18), condition (20b), explicit formula (22c) can fail under such assumptions. In particular, the hypothesis of [5, Remark 3] can fail under the assumption of local monotonicity of $g$.

\section{Explicit Form of $\tau$-Vanishing Shadow Price}

Previously, we examined two transversality conditions (11a) and (11b); consider the two conditions

$$
\begin{aligned}
\lim _{t \rightarrow \infty}\left\|\psi^{0}(t) A_{0}(t)\right\|_{\mathbb{X}} & =0, \\
\liminf _{n \rightarrow \infty}\left\|\psi^{0}\left(\tau_{n}\right) A_{0}\left(\tau_{n}\right)\right\|_{\mathbb{X}} & =0 .
\end{aligned}
$$

Lemma 1 For each solution $\left(x^{0}, u^{0}, \lambda^{0}, \psi^{0}\right) \in \mathfrak{Y}$, the transversality condition (20b) holds iff $\psi^{0}(0)$ is a partial limit of the sequence $\left(\lambda^{0} I_{0}\left(\tau_{n}\right)\right)_{n \in \mathbb{N}}$.

Proof Note that $\lambda^{0} I_{0}\left(\tau_{n}\right)=\lambda^{0}\left(I_{0}\left(\tau_{n}\right)-I_{0}(0)\right)=\psi^{0}(0) A_{0}(0)-\psi^{0}\left(\tau_{n}\right) A_{0}\left(\tau_{n}\right)=\psi^{0}(0)$. Passing to the limit, we obtain what was required; $\lambda^{0} \neq 0$ by virtue of (5a).

Note that the transversality condition (20b) can fail for the $\tau$-vanishing Lagrange multiplier $\left(1, \psi^{0}\right)$ (see Example 4).

Lemma 2 If a nontrivial Lagrange multiplier $\left(1, \psi^{0}\right)$ associated with $\left(x^{0}, u^{0}\right)$ satisfies the transversality condition (20b), then this multiplier is $\tau$-vanishing.

Indeed, there exists $\tau^{\prime} \subset \tau$, for which $\psi^{0}\left(\tau_{n}^{\prime}\right) A_{0}\left(\tau_{n}^{\prime}\right) \rightarrow 0_{\mathbb{X}}$. Then $\psi^{0}(0)-I_{0}\left(\tau_{n}^{\prime}\right)=$ $\psi^{0}\left(\tau_{n}^{\prime}\right) A_{0}\left(\tau_{n}^{\prime}\right) \rightarrow 0_{\mathbb{X}}$, and $I_{0}\left(\tau_{n}^{\prime}\right) \rightarrow \psi^{0}(0)$. Set $\psi_{n}(t) \triangleq\left(I_{0}\left(\tau_{n}^{\prime}\right)-I_{0}(t)\right) A_{0}^{-1}(t)$. Then $\psi_{n}\left(\tau_{n}^{\prime}\right)=0_{\mathbb{X}}, \psi^{0}(0)-\psi_{n}(0)=\psi^{0}(0)-I_{0}\left(\tau_{n}^{\prime}\right) \stackrel{(6 \mathrm{~d})}{=} \psi^{0}\left(\tau_{n}^{\prime}\right) A_{0}\left(\tau_{n}^{\prime}\right) \rightarrow 0_{\mathbb{X}}$. The proof is completed by virtue of the uniform on each compact convergence $\psi_{n} \rightarrow \psi^{0}$.

\subsection{Uniformity in Initial Conditions}

Theorem 3 Assume that conditions $\left(\mathbf{u}_{\sigma}\right),(\mathbf{f g}),(\tau)$ hold. Let one of the two conditions

$$
\begin{aligned}
\text { either } \exists I_{*} \triangleq \lim _{n \rightarrow \infty, \xi \rightarrow 0 \mathbb{X}} I_{\xi}\left(\tau_{n}\right) \in \mathbb{X} ; \\
\text { or } \exists \iota_{*} \triangleq \lim _{n \rightarrow \infty, \xi \rightarrow 0_{\mathbb{X}}} \frac{I_{\xi}\left(\tau_{n}\right)}{\left\|I_{\xi}\left(\tau_{n}\right)\right\|_{\mathbb{X}}} \in \mathbb{X}, \lim _{n \rightarrow \infty, \xi \rightarrow 0_{\mathbb{X}}}\left\|I_{\xi}\left(\tau_{n}\right)\right\|_{\mathbb{X}}=\infty
\end{aligned}
$$

hold. 
Then, there exists a $\tau$-vanishing multiplier $\left(\lambda^{0}, \psi^{0}\right) \in \Lambda$. Moreover, this multiplier satisfies for all $T \in \mathbb{T}$ the corresponding formula of

$$
\begin{aligned}
& \lambda^{0}=1, \quad \psi^{0}(T) \triangleq\left(I_{*}-\int_{0}^{T} \frac{\partial g}{\partial x}\left(t, x^{0}(t), u^{0}(t)\right) A_{0}(t) d t\right) A_{0}^{-1}(T) ; \\
& \lambda^{0}=0, \quad \psi^{0}(T) \triangleq \iota_{*} A_{0}^{-1}(T) .
\end{aligned}
$$

Corollary 8 Assume conditions $\left(\mathbf{u}_{\sigma}\right),(\mathbf{f g}),(\tau)$ hold. Let the limit

$$
\lim _{t \rightarrow \infty, \xi \rightarrow 0 \mathbb{X}} I_{\xi}(t)=\int_{0}^{\infty} \frac{\partial g}{\partial x}\left(t, x^{0}(t), u^{0}(t)\right) A_{0}(t) d t
$$

be well-defined and finite.

Then, the pair $\left(x^{0}, u^{0}\right)$ is normal and there exists a unique $\tau$-vanishing multiplier $\left(\lambda^{0}, \psi^{0}\right) \in \Lambda$. Moreover, for every solution $\left(x^{0}, u^{0}, \lambda^{0}, \psi^{0}\right)$ of core relations of the Maximum Principle (4a)-(4c) and (5b), the following conditions are equivalent:

(1) Its Lagrange multiplier $\left(\lambda^{0}, \psi^{0}\right)$ is $\tau$-vanishing;

(2) The transversality condition (20b) holds;

(3) The transversality condition (20a) holds;

$$
\lambda^{0} \triangleq 1, \quad \psi^{0}(T) \triangleq \int_{T}^{\infty} \frac{\partial g}{\partial x}\left(t, x^{0}(t), u^{0}(t)\right) A_{0}(t) d t A_{0}^{-1}(T) \quad \forall T \in \mathbb{T} .
$$

Case $(b)$ of Theorem 3 is shown in Corollary 6; case (a) will be proved below together with Proposition 5.

In contrast with $(a)$, case $(b)$ expresses the $\tau$-vanishing Lagrange multiplier of a degenerate problem; the author has no knowledge of similar results. Together, these two cases allow to solve problem (1a)-(1b) through relations of the Maximum Principle regardless of its degeneracy (see Example 3).

Note that formula (22a) uses the information about the subsequence of $\tau$. This allows us to find a sequence $\tau$ such that a $\tau$-optimal control exists (see Example 2).

The alternative $(21 \mathrm{a}) \Rightarrow(22 \mathrm{a})$ vs. $(21 \mathrm{~b}) \Rightarrow(22 \mathrm{~b})$ is sufficiently convenient. The need for existence of the limit as $n \rightarrow \infty$ in one of relations (21a), (21b) can always be satisfied if we consider a subsequence. However, Example 4 shows that a unique non-degenerate $\tau$-vanishing multiplier does not necessarily satisfy (20b), even if the improper integral from (22c) converges absolutely. Then, the limit in (21a) (or (21b)) should exist not only for $\xi=0_{\mathbb{X}}$, but also as $\xi \rightarrow 0_{\mathbb{X}}$. In some cases, it is provided outright, for example, if the functions $f$ and $g$ are linear by $x$ (see Example 3), or (see Example 5) by the following remark:

Corollary 9 Assumptions of Theorem 3 hold for a subsequence $\tau^{\prime} \subset \tau$ if one of the assumptions either the functions $f$, $g$ are linear with respect to $x$,

$$
\begin{gathered}
\text { or } \lim _{n \rightarrow \infty, \xi \rightarrow 0_{\mathbb{X}}}\left(I_{\xi}\left(\tau_{n}\right)-I_{0}\left(\tau_{n}\right)\right)=0_{\mathbb{X}}, \\
\text { or } \quad \lim _{n \rightarrow \infty, \xi \rightarrow 0_{\mathbb{X}}} \frac{I_{\xi}\left(\tau_{n}\right)-I_{0}\left(\tau_{n}\right)}{\left\|I_{0}\left(\tau_{n}\right)\right\|_{\mathbb{X}}}=0_{\mathbb{X}},
\end{gathered}
$$

is satisfied. 
Let us finish the proof of Theorem 3. Substituting $T=0$ into (22a) yields $I_{*}=$ $\psi^{0}(0)$; then, Lemma 1 implies

Lemma 3 A solution $\left(x^{0}, \psi^{0}\right)$ of $(4 \mathrm{a})-(4 \mathrm{~b})$ given by formula (22a) satisfies (20b) iff $I_{*}$ is a partial limit of the sequence $\left(\lambda^{0} I_{0}\left(\tau_{n}\right)\right)_{n \in \mathbb{N}}$,

Proposition 5 Assume conditions $\left(\mathbf{u}_{\sigma}\right),(\mathbf{f g}),(\tau)$ hold. Let the map $I_{0}$ be bounded and let

$$
\lim _{\xi \rightarrow 0_{\mathbb{X}}}\left\|I_{\xi}-I_{0}\right\|_{C}=0 .
$$

Then, the pair $\left(x^{0}, u^{0}\right)$ is normal and

(1) there exists a $\tau$-vanishing multiplier $\left(1, \psi^{0}\right) \in \Lambda$ such that transversality condition (20b) holds;

(2) a Lagrange multiplier $\left(\lambda^{0}, \psi^{0}\right)$ associated with $\left(x^{0}, u^{0}\right)$ is $\tau$-vanishing iff the transversality condition (20b) holds.

(3) a limit point $I_{*} \in \mathbb{X}$ of the sequence $\left(I_{0}\left(\tau_{n}\right)\right)_{n \in \mathbb{N}}$ corresponds to each $\tau$-vanishing multiplier $\left(\lambda^{0}, \psi^{0}\right) \in \Lambda$, and a $\tau$-vanishing multiplier $\left(\lambda^{0}, \psi^{0}\right) \in \Lambda$ corresponds to each limit point $I_{*} \in \mathbb{X}$ of the sequence $\left(I_{0}\left(\tau_{n}\right)\right)_{n \in \mathbb{N}}$. This bijection is given by $(22 \mathrm{a})$.

Proof By Theorem 2, a $\tau$-vanishing multiplier exists; by Remark 3, any $\tau$-vanishing multiplier $\left(\lambda^{0}, \psi^{0}\right)$ satisfies $\lambda^{0}>0$; moreover, by $(5 b)$, if $\left(\lambda^{0}, \psi^{0}\right) \in \Lambda$, then $\lambda^{0}=1$. Now, by (9a), we have

$$
\psi^{0}(0)=\lim _{n \rightarrow \infty} \lambda^{n} I_{\xi_{n}}\left(\tau_{n}^{\prime}\right)=\lambda^{0} \lim _{n \rightarrow \infty, \xi \rightarrow 0 \mathbb{X}} I_{\xi}\left(\tau_{n}^{\prime}\right)=\lambda^{0} I_{*},
$$

and from Lemmas 1 and 3, we obtain (20b) and (22a). The inverse is true by virtue of Lemma 2.

\subsection{Uniformity by Control}

Formulations of the preceding section can be expressed in another form. By varying, instead of the initial point $\xi$, the control $u$ near $u^{0}$, we pass from $x_{\xi}, A_{\xi}, I_{\xi}$ to $x^{u}, A^{u}, I^{u}$.

Fix pair $(p, v) \in(0, \infty) \times B_{\text {loc }}\left(\mathbb{T}, \mathbb{R}_{>0}\right)$. As in Remark 3 , we have

Corollary 10 Assume conditions $(\mathbf{u}),(\mathbf{f g}),(\tau)$. If for the control $u^{0}$ and some subsequence $\tau^{\prime} \subset \tau$ we have

$$
\limsup _{n \rightarrow \infty, \varrho\left(\eta, u^{0} ; \widetilde{\mathcal{L}}_{\nu}^{p}\left(\left[0, \tau_{n}^{\prime}\right], \mathbb{U}\right)\right) \rightarrow 0}\left\|\int_{0}^{\tau_{n}^{\prime}} \frac{\partial g}{\partial x}\left(t, x^{\eta}(t), u(t)\right) A^{\eta}(t) d t\right\|_{\mathbb{X}}<\infty,
$$

then the pair $\left(x^{0}, u^{0}\right)$ is normal; there exists a $\tau$-vanishing multiplier $(1, \psi) \in \Lambda$.

Proof By Remark 2, there exist a $\tau^{\prime}$-vanishing multiplier $\left(\lambda^{0}, \psi^{0}\right)$ and sequences $\tau^{\prime \prime} \subset$ $\tau^{\prime},\left(x^{n}, \eta^{n}, \lambda^{n}, \psi^{n}\right)_{n \in \mathbb{N}}$ such that Remark 1 and $\varrho\left(\eta^{n}, u^{0} ; \widetilde{\mathcal{L}}_{v}^{p}(\mathbb{T}, \mathbb{U})\right) \rightarrow 0$ hold. Then, 
$\varrho\left(\eta^{n}, u^{0} ; \widetilde{\mathcal{L}}_{v}^{p}\left(\left[0, \tau_{n}^{\prime \prime}\right], \mathbb{U}\right)\right) \rightarrow 0$; therefore, $\left(I^{\eta^{n}}\left(\tau_{n}^{\prime}\right)\right)_{n \in \mathbb{N}}$ is bounded by the assumption of the corollary. But $\lambda^{n} I^{\eta^{n}}\left(\tau_{n}^{\prime \prime}\right) \rightarrow \psi^{0}(0)$, thus $\lambda^{0}>0$. Now $\left(1, \psi^{0} / \lambda^{0}\right)$ is a $\tau$-vanishing multiplier.

Corollary 11 Assume conditions $(\mathbf{u}),(\mathbf{f g}),(\tau)$ hold. Let $I_{0}$ be bounded and let

$$
\left\|I_{0}-I^{\eta}\right\|_{C\left(\left[0, \tau_{n}\right], \mathbb{X}\right)} \rightarrow 0 \quad \text { as } \quad n \rightarrow \infty, \varrho\left(\eta, u^{0} ; \widetilde{\mathcal{L}}_{v}^{p}\left(\left[0, \tau_{n}\right], \mathbb{U}\right) \rightarrow 0 .\right.
$$

Then, the pair $\left(x^{0}, u^{0}\right)$ is normal, and

(1) a $\tau$-vanishing multiplier $\left(1, \psi^{0}\right) \in \Lambda$ corresponds to each partial limit $I_{*} \in \mathbb{X}$ of the sequence $\left(I_{0}\left(\tau_{n}\right)\right)_{n \in \mathbb{N}}$ by formula (22a);

(2) all such multipliers satisfy transversality condition (20b).

Proof Let $I_{*}$ be the limit of $\left(I_{0}\left(\tau_{n}^{\prime}\right)\right)_{n \in \mathbb{N}}$ for certain $\tau^{\prime} \subset \tau$. Then, by Corollary 10 , there exists a $\tau^{\prime}$-vanishing multiplier $\left(1, \psi^{0}\right)$ such that $\psi^{0}(0)=\lambda^{0} \lim _{n \rightarrow \infty} I^{\eta^{n}}\left(\tau_{n}^{\prime \prime}\right)=$ $\lim _{n \rightarrow \infty} I^{\eta^{n}}\left(\tau_{n}^{\prime \prime}\right)$ for some $\tau^{\prime \prime} \subset \tau^{\prime}$. By assumptions of the corollary, we obtain $\psi^{0}(0)=$ $\lambda^{0} I_{*}$. But this, by Lemma 1 , is equivalent to (20b). Substituting $\psi^{0}(0)=\lambda^{0} I_{*}$ into $(6 d)$, we obtain (22a).

Repeating the proof of Corollary 10, but, this time, using (13), we get

Corollary 12 Assume conditions $(\mathbf{u}),(\mathbf{f g}),(\tau)$ hold. Let for some $\iota_{*} \in \mathbb{X}$ there be

$$
\frac{I^{\eta}\left(\tau_{n}\right)}{\left\|I^{\eta}\left(\tau_{n}\right)\right\|_{\mathbb{X}}} \rightarrow \iota_{*},\left\|I^{\eta}\right\|_{C\left(\left[0, \tau_{n}\right], \mathbb{X}\right)} \rightarrow \infty \quad \text { as } n \rightarrow \infty, \varrho\left(\eta, u^{0} ; \widetilde{\mathcal{L}}_{v}^{p}\left(\left[0, \tau_{n}\right], \mathbb{U}\right) \rightarrow 0\right.
$$

Then, for the pair $\left(x^{0}, u^{0}\right)$, there exists a degenerate $\tau$-vanishing multiplier $\left(0, \psi^{0}\right)$ such that condition (20b) and formula (22b) hold.

\subsection{Conditions Guaranteeing Convergence to $I_{*}$}

Let us consider the conditions on the system that are both sufficiently easy to check and sufficient to make use of Corollary 8.

Proposition 6 Assume conditions $\left(\mathbf{u}_{\sigma}\right),(\mathbf{f g}),(\tau)$ hold. For certain measurable functions $F \in \mathcal{L}_{\text {loc }}^{1}(\mathbb{T}, \mathbb{L}), G \in \mathcal{L}_{\text {loc }}^{1}(\mathbb{T}, \mathbb{X})$, a summable function $\omega \in \mathfrak{L}^{1}(\mathbb{T}, \mathbb{T})$, let

$$
G(t) \succcurlyeq \frac{\partial g}{\partial x}\left(t, x, u^{0}(t)\right) \succcurlyeq-G(t), \quad F(t) \succcurlyeq \frac{\partial f}{\partial x}\left(t, x, u^{0}(t)\right) \succcurlyeq-F(t),
$$

$$
\left\|G(t) B_{*}(t)\right\|_{\mathbb{X}} \leq \omega(t)
$$

for all $(t, x) \in \mathbb{T} \times \mathbb{X}$, where $B_{*}$ is a matrix solution of

$$
\dot{B}_{*}(t)=F(t) B_{*}(t), \quad B_{*}(0)=1_{\mathbb{L}} \quad \forall \text { a.a.t } \in \mathbb{T} .
$$

Then, the result of Corollary 8 holds. 
Proof For each $B=\left(b_{i j}\right)_{i, j \in \overline{1, m}} \in \mathbb{L}, C=\left(c_{i}\right)_{i \in \overline{1, m}} \in \mathbb{X}$, let us introduce

$$
B^{\sharp} \triangleq\left(\left|b_{i j}\right|\right)_{i, j \in \overline{1, m}} \in \mathbb{L}, C^{\sharp} \triangleq\left(\left|c_{i}\right|\right)_{i \in \overline{1, m}} \in \mathbb{X} .
$$

It is easy to see that $B^{\sharp} \succcurlyeq 0_{\mathbb{L}}, C^{\sharp} \succcurlyeq 0_{\mathbb{X}}, B^{\sharp} \succcurlyeq B \succcurlyeq-B^{\sharp}, C^{\sharp} \succcurlyeq C \succcurlyeq-C^{\sharp}$. Moreover, $C^{\sharp} B^{\sharp} \succcurlyeq C B \succcurlyeq-C^{\sharp} B^{\sharp}$ for all $B \in \mathbb{L}, C \in \mathbb{X}$.

Denote by $F_{\xi}(t)$ the matrix $\frac{\partial f}{\partial x}\left(t, x_{\xi}(t), u^{0}(t)\right)$ for all $t \in \mathbb{T}$. Now, for all $\xi \in \mathbb{X}$, we have

$$
F(t) \succcurlyeq F_{\xi}^{\sharp}(t) \succcurlyeq F_{\xi}(t) \succcurlyeq-F_{\xi}^{\sharp}(t) \succcurlyeq-F(t) \quad \forall a . a . t \in \mathbb{T} .
$$

Compare the right-hand sides and the initial conditions of equations (23c), (6b), and equation

$$
\dot{B}_{\xi}(t)=F_{\xi}^{\sharp}(t) B_{\xi}(t), \quad B_{\xi}(0)=1_{\mathbb{L}} .
$$

For solution $B_{\xi}$ by the comparison theorem, we obtain

$$
B_{*}(t) \succcurlyeq B_{\xi}(t) \succcurlyeq A_{\xi}(t) \succcurlyeq-B_{\xi}(t) \succcurlyeq-B_{*}(t) \quad \forall \text { a.a. } t \in \mathbb{T}
$$

in particular, $B_{*}(t) \succcurlyeq A_{\xi}^{\sharp}(t)$.

Now, we have $G(t) B_{*}(t) \succcurlyeq\left(\frac{\partial g}{\partial x}\left(t, x_{\xi}(t), u^{0}(t)\right)\right)^{\sharp} A_{\xi}^{\sharp}(t) \succcurlyeq\left(\dot{I}_{\xi}(t)\right)^{\sharp}$, whence we obtain $G(t) B_{*}(t) \succcurlyeq \dot{I}_{\xi}(t) \succcurlyeq-G(t) B_{*}(t),\left\|\dot{I}_{\xi}(t)\right\|_{\mathbb{X}} \leq\left\|G(t) B_{*}(t)\right\|_{\mathbb{X}} \leq \omega(t)$ for all $\xi \in \varepsilon_{0} \mathbb{D}$, for a.a. $t \in \mathbb{T}$. We have

$$
\begin{array}{r}
\left\|I_{\xi}\right\|_{C} \leq\left\|I_{\xi}\right\|_{C([0, T], \mathbb{X})}+\int_{T}^{\infty} \omega(t) d t, \\
\left\|I_{\xi}-I_{0}\right\|_{C} \leq\left\|I_{\xi}-I_{0}\right\|_{C([0, T], \mathbb{X})}+2 \int_{T}^{\infty} \omega(t) d t .
\end{array}
$$

For each $\varepsilon>0$, it is possible to find $T \in \mathbb{T}$, for which the second summands do not exceed $\varepsilon$, and yet $\left.\left.I_{\xi}\right|_{[0, T]} \rightarrow I_{0}\right|_{[0, T]}$ for $\xi \rightarrow 0_{\mathbb{X}}$. Then all conditions of Corollary 8 hold.

Remark 6 The first condition of (23a) of Proposition 6 could be formally weakened down to

$$
F(t)+m(t) 1_{\mathbb{L}} \succcurlyeq \frac{\partial f}{\partial x}\left(t, x, u^{0}(t)\right) \succcurlyeq-F(t)-m(t) 1_{\mathbb{L}},
$$

for some summable function $m \in \mathfrak{L}^{1}(\mathbb{T}, \mathbb{T})$.

Indeed, consider a number $R=e^{\int_{0}^{\infty} m(\theta) d \theta} \in \mathbb{T}$, a summable function $\omega_{1} \triangleq R \omega$, and a matrix function $F_{1} \triangleq F+m 1_{\mathbb{L}}$. Now, $B_{1}(t) \triangleq e^{\int_{[0, t]} m(\theta) d \theta} B_{*}(t)$ solves the equation $\dot{B}_{1}=F_{1} B_{1}, B_{1}(0)=1_{\mathbb{L}}$ and

$$
\left\|G(t) B_{1}(t)\right\|_{\mathbb{X}}=e^{\int_{[0, t]} m(\theta) d \theta}\left\|G(t) B_{*}(t)\right\|_{\mathbb{X}} \leq e^{\int_{[0, t]} m(\theta) d \theta} \omega(t) \leq R \omega(t)=\omega_{1}(t) .
$$

Thus, under conditions of the remark, all propositions of Proposition 6 hold for $F_{1}, \omega_{1}$ in the place of $F, \omega$. 
Note that conditions of Proposition 6 (taking into account Remark 6) for a smooth control problem without phase restrictions are weaker than conditions $[36,(\mathrm{C} 1)-$ (C3)]. To be more precise, condition $[36,(\mathrm{C} 1)]$ is exactly condition $(\mathbf{u})$, and $[36,(\mathrm{C} 2)]$ is exactly (23b). Condition $[36,(\mathrm{C} 3)]$ requires $\left\|G(t) B_{*}(t) B_{*}^{-1}(\theta)\right\|_{\mathbb{X}} \leq \omega(t)$ for all $t \in$ $\mathbb{T}, \theta \in[0, t]$, while condition (23a) requires this only for $t \in \mathbb{T}, \theta=0$. In particular, in [3, Example 16.1], conditions of [3, Theorem 12.1] and Proposition 6 hold if $\rho>0$, and conditions $[36,(\mathrm{C} 1)-(\mathrm{C} 3)]$ only hold if $\rho>1$.

Corollary 13 Assume conditions $(\mathbf{u}),(\mathbf{f g}),(\tau)$ hold. For a summable function $\omega \in$ $\mathfrak{L}^{1}(\mathbb{T}, \mathbb{T})$ for all $u \in \mathfrak{U}$, let

$$
\left\|\frac{\partial g}{\partial x}\left(t, x^{u}(t), u(t)\right) A^{u}(t)\right\|_{\mathbb{X}} \leq \omega(t)
$$

Then, the pair $\left(x^{0}, u^{0}\right)$ is normal and Corollary 8 holds with exception of uniqueness of the $\tau$-vanishing multipliers; specifically,

(1) Exactly one $\tau$-vanishing multiplier satisfies (5b) and (20b);

(2) Exactly one $\tau$-vanishing multiplier satisfies (5b) and (20a);

(3) Actually, it is the $\tau$-vanishing multiplier $\left(1, \psi^{0}\right) \in \Lambda$; and this multiplier could be obtained by formula (22c).

The results of this corollary can fail if (24) only holds for $u=u^{0}$ (see Example 4).

Proof Note that (24) holds not only for all $u \in \mathfrak{U}$, but also for all $\eta \in \widetilde{\mathfrak{U}}$; then, for all $T \in \mathbb{T}$, we have

$$
\begin{array}{r}
\left\|I^{\eta}\right\|_{C} \stackrel{(24)}{\leq}\left\|I^{\eta}\right\|_{C([0, T], \mathbb{X})}+\int_{T}^{\infty} \omega(t) d t, \\
\left\|I^{\eta}-I_{0}\right\|_{C} \stackrel{(24)}{\leq}\left\|I^{\eta}-I_{0}\right\|_{C([0, T], \mathbb{X})}+2 \int_{T}^{\infty} \omega(t) d t .
\end{array}
$$

For each $\varepsilon>0$, there exists a $T \in \mathbb{T}$ such that the second summands do not exceed $\varepsilon / 2$. Let us construct the $\tau$-vanishing multiplier $\left(\lambda^{0}, \psi^{0}\right) \in \Lambda$ by a limit of sequences from Remark 1, but Proposition 1 implies $\left.\left.I^{\eta}\right|_{[0, T]} \rightarrow I_{0}\right|_{[0, T]}$ for $\eta \rightarrow \widetilde{u}^{0}$. Hence, $\left\|I^{n}-I_{0}\right\|_{C} \rightarrow 0$, and $I_{0}$ is bounded. Since $\psi^{n}\left(\tau_{n}^{\prime}\right)=0_{\mathbb{X}}$, we know that (20b) holds for $\psi^{0}$.

From (24) for $u=u^{0}$, we see that for any unboundedly increasing sequence of times $v$, the sequence $\left(I_{0}\left(v_{n}\right)\right)_{n \in \mathbb{N}}$ is fundamental and thus it has the limit point $I_{*}$. Since this is correct for any unboundedly increasing sequence of times, $I_{0}(t) \rightarrow I_{*}$ as $t \rightarrow \infty$. Now, Lemma 1 yields item 2). Finally, Lemma 3 implies (22c).

The formula (22c) was obtained by Kryazhimskii and Aseev under easily checked assumptions on growth of functions $f, g$ and their derivatives (see stationary case in [3, Theorem 12.1], [5, Theorem 4] and non-stationary case in [7, Theorem 1]). This condition generalizes (see [3, Section 16], [7]) a number of transversality conditions; in particular, it is more general than the conditions that were obtained for linear systems in [9]. 
From conditions of [4, Theorem 2], [3, Theorem 12.1], and [2, Theorem 1], it follows that for some $\alpha, \beta>0$ and for all admissible controls $u \in \mathfrak{U}$, all trajectories $x$, and all fundamental matrices $A$, the following inequality holds:

$$
\left\|\frac{\partial g}{\partial x}(t, x(t), u(t))\right\|\|A(t)\| \leq \beta e^{-\alpha t} \quad \forall t \in \mathbb{T}
$$

(see, for example, [3, (A5)-(A7)]). This is stronger than the conditions of Corollary 13. In paper [7], it was actually assumed that (25) holds for $x=x_{\xi}, A=$ $A_{\xi}, u=u^{0}$ if $\xi$ is sufficiently small. Aseev and Veliov also proved necessity of (22c) in paper [8]. The assumptions on $f, g$ from [8] are weaker than (25), but from its assumptions it follows that conditions in Corollary 8 hold, and the improper integral in (22c) converges in the Lebesgue sense, converges absolutely. However, it is worth noting that $[7,8]$ use a more general definition of optimality (the locally weakly overtaking optimality). In addition, condition (25) can be verified by calculating the characteristic Lyapunov exponents of the system of the Maximum Principle, see [3, Section 12], [4, Section 3], and [7, Section 5].

Observe that (25) are characteristic of economic problems with exponentially decreasing discount factor; however, one could consider other non-subexponential discount factors (see $[20,21,46,47]$ ). Example 5 exhibits the solution of a problem with such discount factor.

For economic problems with decreasing discount factor (specifically, for (19)) in [5, Theorem 4], sufficiently broad conditions for applicability of formula (22c) were obtained. It turns out that it is sufficient to connect (see [5, (A4)] and (26)) the growth of $I^{u}$ with the growth of $J^{u}$. In contrast with the results of [7] or Corollary 13, the finiteness of the optimal result on the optimal trajectory is required, and it is not guaranteed that the $\tau$-vanishing multiplier is unique. Let us transfer this result of [5, Theorem 4] from case (19) to general non-stationary system (1a)-(1b).

Corollary 14 Assume conditions $(\mathbf{u}),(\mathbf{f g}),(\tau)$ hold. Let there exist the finite limit $\lim _{n \rightarrow \infty} J^{u^{0}}\left(\tau_{n}\right)$. Let a functions $\omega_{0}, \omega_{\infty} \in C(\mathbb{T}, \mathbb{T})$ satisfy $\omega_{0}(0)=0, \omega_{\infty}\left(\tau_{n}\right) \rightarrow 0$ as $n \rightarrow$ $\infty$. For all $\eta \in \widetilde{\mathfrak{U}}$ from some $\mathcal{L}_{v}^{p}(\mathbb{T}, \mathbb{U})$-neighborhood $O_{v}^{p}$ of the control $u^{0}$ for all $k, n \in \mathbb{N}, k<n$, let there be

$$
\left\|\int_{\tau_{k}}^{\tau_{n}} \frac{\partial g}{\partial x}\left(t, x^{\eta}(t), u(t)\right) A^{\eta}(t) d t\right\|_{\mathbb{X}} \leq \omega_{\infty}\left(\tau_{k}\right)+\omega_{0}\left(\left|J^{\eta}\left(\tau_{n}\right)-J^{\eta}\left(\tau_{k}\right)\right|\right) .
$$

Then, the pair $\left(x^{0}, u^{0}\right)$ is normal, the limit $I_{*}=\lim _{n \rightarrow \infty} I^{0}\left(\tau_{n}\right) \in \mathbb{X}$ is well-defined, and

(1) Exactly one multiplier satisfies (5b) and (20b);

(2) Actually, it is the $\tau$-vanishing multiplier $\left(1, \psi^{0}\right) \in \Lambda$; and this multiplier could be obtained by formula (22c).

Proof There exists a sequence $\left(s_{k}\right)_{k \in \mathbb{N}} \downarrow 0$ such that for all $k, n \in \mathbb{N}, k<n$, we have $\left|J^{u^{0}}\left(\tau_{n}\right)-J^{u^{0}}\left(\tau_{k}\right)\right|<s_{k}$. Substituting $u=u^{0}$ into (26) yields the existence of the finite limit $I_{*}=\lim _{n \rightarrow \infty} I^{0}\left(\tau_{n}\right)$. Now, as in the proof of Corollary 13, we show that there exists the unique solution from $\mathfrak{Y}$ that satisfies $(20 b)$ and that for it, accurately to a positive factor, the formula (22a) is correct. It only remains to prove that the pair defined by (22a) is a $\tau$-vanishing multiplier. 
By Theorem 2, for this problem there exists the $\tau$-vanishing multiplier $\left(\lambda^{0}, \psi^{0}\right) \in$ $\Lambda$ that was constructed as the uniform limit of sequence $\left(x^{n}, \eta^{n}, \lambda^{n}, \psi^{n}\right)_{n \in \mathbb{N}} \in \widetilde{\mathfrak{Y}}^{\mathbb{N}}$ from Remark 1. Passing to the subsequence $\tau^{\prime} \subset \tau$ if necessary, we may assume that the sequence of $\eta^{n}$ is included in the closure of $O_{v}^{p}$; then, (26) holds for each $\eta^{n}$. The function $\omega_{0}$ can be considered monotonic without loss of generality. Then, using the triangle inequality twice and, by the inequality $\widetilde{J}^{\eta^{n}}\left(\tau_{n}\right)-J^{u^{0}}\left(\tau_{n}\right) \geq 0$, for all $k, n \in \mathbb{N}, k<n$, we have the following:

$$
\begin{aligned}
\left\|I^{\eta^{n}}\left(\tau_{n}\right)-I^{\eta^{n}}\left(\tau_{k}\right)\right\| & \stackrel{(26)}{\leq} \omega_{\infty}\left(\tau_{k}\right)+\omega_{0}\left(\left|\widetilde{J}^{\eta^{n}}\left(\tau_{n}\right)-\widetilde{J}^{\eta^{n}}\left(\tau_{k}\right)\right|\right) \\
\leq & \omega_{\infty}\left(\tau_{k}\right)+\omega_{0}\left(\widetilde{J}^{\eta^{n}}\left(\tau_{n}\right)-J^{u^{0}}\left(\tau_{n}\right)+\mid \widetilde{J}^{\eta^{n}}\left(\tau_{k}\right)\right. \\
& \left.-J^{u^{0}}\left(\tau_{k}\right)|+| J^{u^{0}}\left(\tau_{n}\right)-J^{u^{0}}\left(\tau_{k}\right) \mid\right) \\
& \stackrel{(\widetilde{2})}{\leq} \omega_{\infty}\left(\tau_{k}\right)+\omega_{0}\left(\gamma_{n}^{2}+\left|\widetilde{J}^{\eta^{n}}\left(\tau_{k}\right)-J^{u^{0}}\left(\tau_{k}\right)\right|+s_{k}\right), \\
\left\|I^{\eta^{n}}\left(\tau_{n}\right)-I^{0}\left(\tau_{n}\right)\right\| \|_{\mathbb{X}} \leq & \left\|I^{\eta^{n}}\left(\tau_{n}\right)-I^{\eta^{n}}\left(\tau_{k}\right)\right\| \mathbb{X}+|| I^{\eta^{n}}\left(\tau_{k}\right)-I^{0}\left(\tau_{k}\right)\left\|\mathbb{X}+|| I^{0}\left(\tau_{k}\right)-I^{0}\left(\tau_{n}\right)\right\|_{\mathbb{X}} \\
\stackrel{(26)}{\leq}|| I^{\eta^{n}}\left(\tau_{k}\right)-I^{0}\left(\tau_{k}\right) \| \mathbb{X}+2 \omega_{\infty}\left(\tau_{k}\right)+\omega_{0}\left(s_{k}\right) & \\
& +\omega_{0}\left(\gamma_{n}^{2}+\left|\widetilde{J}^{\eta^{n}}\left(\tau_{k}\right)-J^{u^{0}}\left(\tau_{k}\right)\right|+s_{k}\right) .
\end{aligned}
$$

Since $I^{\eta}, \widetilde{J}^{\eta}$ converges to $I_{0}, J^{u^{0}}$ uniformly on every compact and by definition of $\gamma_{n}$, passing to the limit as $n \rightarrow \infty$, we have

$$
\limsup _{n \rightarrow \infty}\left\|I^{\eta^{n}}\left(\tau_{n}\right)-I^{0}\left(\tau_{n}\right)\right\|_{\mathbb{X}} \leq 2 \omega_{\infty}\left(\tau_{k}\right)+2 \omega_{0}\left(s_{k}\right) .
$$

By definitions of $\omega_{0}, \omega_{\infty}$, and $s_{k}$, passing to the limit as $k \rightarrow \infty$, we see that $I^{\eta^{n}}\left(\tau_{n}\right)-$ $I^{0}\left(\tau_{n}\right) \rightarrow 0_{\mathbb{X}}$.

Now, by Remark 1 , we have $\lambda^{n} I^{0}\left(\tau_{n}\right) \rightarrow \psi^{0}(0)$. Since $I^{0}\left(\tau_{n}\right) \rightarrow I_{*}$, we know that $\lambda^{0}>0$ and $\lambda^{0} \psi^{0}(0)=I_{*}$ hold. By dividing this $\left(\lambda^{0}, \psi^{0}\right)$ on $\lambda^{0}$, we obtain (22a).

\section{Examples}

Example 1 The feature of [36, Example 10.2] lies in the fact that transversality condition (11a) fails to give any information that could help us in determining the unique Lagrange multiplier. Let us show that the definition of a $\tau$-vanishing multiplier allows us to do it.

$$
\dot{x}=u x, x(0)=1, u \in[1 / 2,1], \quad J^{u}(T) \triangleq \int_{0}^{T} x e^{-2 t} d t \stackrel{T \rightarrow \infty}{\rightsquigarrow} \max .
$$

Here, $\mathcal{H}=u \psi x+e^{-2 t} \lambda x$ and $\dot{\psi}=-u \psi-e^{-2 t} \lambda$. Then, $A=x, I^{u}=J^{u}$; consider $F=1, G=e^{-2 t}, \omega(t)=e^{-t}$. By Proposition 6 , there exists the unique $\tau$-vanishing multiplier. Substituting it into $\mathcal{H}$, we obtain $\mathcal{H}\left(x^{0}(t), t, u^{0}(t), \lambda^{0}, \psi^{0}(t)\right)=u^{0} \lambda^{0}\left(I_{*}-\right.$ $\left.J^{u^{0}}(t)\right)+e^{-2 t} \lambda x^{0}(t)$; now, from $(4 \mathrm{c})$, we have $u^{0} \equiv 1, I_{*}=J^{u^{0}}(+\infty)=1$; then, $\psi^{0}(0)=\lambda^{0}=1$, it is a unique $\tau$-vanishing multiplier. (Of course, in this example, the control $u^{0}$ is easily found in view of the monotonicity of $f, g$ and Corollary 7$)$. 
The alternative $(21 \mathrm{a}) \Rightarrow(22 \mathrm{a})$ versus $(21 \mathrm{~b}) \Rightarrow(22 \mathrm{~b})$ allows us to effectively reduce an optimal problem to the boundary problem of relations of the Maximum Principle. The only obstacle is the uniformity of limits in (21a) and (21b). In some cases, the uniformity of these limits is trivial, for example, when the functions $f$ and $g$ are linear by $x$. Thus, such problems are easy to solve. Let us demonstrate this by the following example:

\section{Example 2}

$$
\dot{x}=y, \quad \dot{y}=-x+u, \quad x(0)=1, \quad y(0)=0, \quad u \in[-1,1], \quad \int_{0}^{T} y d t \stackrel{T \rightarrow \infty}{\sim} \max
$$

Here, for all $t, T \in \mathbb{T}, \xi \in \mathbb{X}$, we have

$$
\begin{aligned}
A_{\xi}(t) & =\left(\begin{array}{cc}
\cos t & \sin t \\
-\sin t & \cos t
\end{array}\right), I_{\xi}(T)=(\cos T-1, \sin T), \\
I_{0}(t) A_{0}^{-1}(T) & =(\cos (t-T)-\cos T, \sin (t-T)+\sin T) .
\end{aligned}
$$

Now, because $I_{\xi}$ is $2 \pi$-periodic, for any sequence $\left(\tau_{n}\right)_{n \in \mathbb{N}}$ there exists a $\varsigma \in[0,2 \pi]$ and subsequence $\tau^{\prime} \subset \tau$ such that $I_{\xi}\left(\tau_{n}^{\prime}\right) \rightarrow I_{0}(\varsigma)$, whence, by Theorem 3 ,

$$
\begin{gathered}
\psi^{0}(T)=\left(I_{0}(\varsigma)-I_{0}(T)\right) A_{0}^{-1}(T)=(\cos (\varsigma-T)-1, \sin (\varsigma-T)) \\
u^{0}(T)=\arg \max _{u \in[-1,1]}(\cos (\varsigma-T)-1, \sin (\varsigma-T))\left(\begin{array}{l}
0 \\
u
\end{array}\right)=\arg \max _{u \in[0,1]} \sin (\varsigma-T) u, \text { i.e. } \\
u^{0}(T)=\operatorname{sgn} \sin (\varsigma-T) \quad \forall \text { a.a. } T \in \mathbb{T} .
\end{gathered}
$$

Observe that the proposed approach finds, first of all, $\tau$-optimal controls. Indeed, let the sequence $\tau$ be given. Express each $\tau_{n}$ in the form $\tau_{n}=2 \pi k_{n}+\sigma_{n}$, where $\sigma_{n} \in$ $[0,2 \pi)$. Substituting each limit point $\zeta$ of the sequence $\left(\sigma_{n}\right)_{n \in \mathbb{N}}$ into $(22 \mathrm{a})$ yields all corresponding $\tau$-vanishing multipliers; moreover, formula (27) yields all prospective $\tau$-optimal controls.

It is easy to check (see [41]) that any control of form (27) is uniformly weakly overtaking optimal, thus each of them is $\tau$-optimal for its sequence $\tau$. Then, there exists a $\tau$-optimal control if there exists a limit of the sequence of $I_{0}\left(\sigma_{n}\right)$.

Also observe that this example specifies why it is impossible to replace transversality condition (20b) in Proposition 5 with the stronger one (20a).

Example 3 Theorem 3 allows, in some circumstances, to find optimal solutions for degenerate problems in the way it is done for nondegenerate. Let us show this. Consider the modification of the well-known Halkin's example [23] (see also [33, Example 5.1], [6, Example 1])

$$
\dot{x}=u x, \quad x(0)=1, \quad \int_{0}^{T}(1-u) x d t \stackrel{T \rightarrow \infty}{\rightarrow} \max , \quad u \in[\alpha, \beta](\alpha \leq \beta) .
$$

Let there exist a weakly uniformly overtaking optimal control in this problem, then, for some sequence $\tau$, this control is $\tau$-optimal. 
Here, $A_{\xi}(T)=x^{0}(T)$ and $I_{\xi}(T)=J^{u^{0}}(T)$. Passing, if necessary, from $\tau$ to its subsequence, we face one of the three cases:

(A) $J^{u^{0}}\left(\tau_{n}\right) \rightarrow+\infty$. From Theorem 3 (b) $\iota_{*}=1, \lambda=0, H[T]=u^{0}, u^{0} \equiv \beta$; if we substitute this into $J^{u^{0}}(T)$, we will obtain $0 \leq \beta<1$.

(B) $J^{u^{0}}\left(\tau_{n}\right) \rightarrow-\infty$; similarly, we have $u^{0} \equiv \alpha>1$.

(C) $J^{u^{0}}\left(\tau_{n}\right) \rightarrow I_{*} \in \mathbb{R}$. Here, by Theorem 3, from (21a) follows (22a); in particular $\lambda^{0}=1$. Consider $R(t) \triangleq I_{*}-J^{u^{0}}(t)-x^{0}(t)$. Then

$$
\begin{aligned}
H\left(x^{0}, t, u, \lambda^{0}, \psi^{0}\right) & =\left(I_{*}-J^{u^{0}}(t)\right) A^{-1}(t) u x^{0}(t)+(1-u) x^{0}(t) \\
& =\left(I_{*}-J^{u^{0}}(t)-x^{0}(t)\right) u+x^{0}(t)=R(t) u+x^{0}(t),
\end{aligned}
$$

Thus, $u^{0}(t)$ is defined by the sign of $R(t)$. Since $\dot{R}(t)=-x^{0}(t)<0$, there is at most one switching point. Then, there exist $T \in \mathbb{T}, p \in\{\alpha, \beta\}$ such that $u^{0}(t)=p$ for a.a. $t>T$. Without loss of generality, either $T=0$, or $R(T)=0$. Moreover, the boundedness of $I_{*}-J^{u^{0}}\left(\tau_{n}\right)$ guarantees that either $p<0$ or $p=1$.

The first case: if $p<0$; then, $x^{0}\left(\tau_{n}\right) \rightarrow 0$ as $n \rightarrow \infty$. Therefore $R\left(\tau_{n}\right) \rightarrow 0$ as $n \rightarrow$ $\infty$. The function $R$ is decreasing, whence $R(t)>0$ for all $t>0$. Thus, $T=0$ and $u^{0} \equiv \beta=p<0$.

The second case: if $p=1$; then $u^{0}(t)=1$ for a.a. $t>T$. Thus, $I_{*}=J^{u^{0}}(t)$ for $t \geq T$, i.e., $R(t)=-x^{0}(t)<0$. Since $R(T) \neq 0$, we have $T=0$. Thus, $u^{0} \equiv \alpha=p=1$.

Collecting all cases, we obtain $u^{0} \equiv \alpha$ for $\alpha \geq 1$, and $u^{0} \equiv \beta$ for $\beta<1$.

Checking this, we show the above controls are indeed $\tau$-optimal (moreover, even uniformly overtaking optimal) control in this problem. Consequentially, the problem has no $\tau$-optimal (and, therefore, no weakly uniformly overtaking optimal) control if $\alpha<1 \leq \beta$. On the other hand, in case $[\alpha, \beta] \triangleq[0,1]$, the control $u^{0} \equiv 0$ is decision horizon optimal (DH-optimal, see [12]). Therefore, in Theorem 2, we could not replace the $\tau$-optimality (weakly uniformly overtaking optimality, uniformly overtaking optimality) with the $D H$-optimality (weekly agreeable, agreeable optimality; [12]).

Example 4 Consider the following problem

$$
\dot{x}=x+u, x(0)=0 ; u \in[0,1], \quad J^{u}(T)=\int_{0}^{T} e^{-2 t} x\left(1-x^{4}\right) d t \stackrel{T \rightarrow \infty}{\sim} \max
$$

Let us show that $x^{0} \equiv 0, u^{0} \equiv 0$ is the optimum. Consider an arbitrary control that differs from $u^{0}$ on the set of nonzero measure and the trajectory $x$ that corresponds to it. Starting from some instance of time, $x$ must be positive, and, moreover, there must exist $T \in \mathbb{T}$ such that $x(T)=1$. Then, $x\left(s_{1}\right) \leq e^{s_{1}-T}$ if $s_{1} \leq T$, and $2 e^{s_{2}-T}-1 \geq$ $x\left(s_{2}\right) \geq e^{s_{2}-T}$ if $s_{2} \geq T$. Now, for $s \leq T$, we have

$$
J^{u}(s) \leq J^{u}(T) \leq \int_{0}^{T} e^{-t-T} d t<e^{-T} \leq e^{-s}
$$


and for $s \geq T$, we have

$$
\begin{aligned}
J^{u}(s) & <e^{-T}+\int_{T}^{s} e^{-2 t} x(t) d t-\int_{T}^{s} e^{-2 t} x^{3}(t) d t \\
& <e^{-T}+\int_{T}^{\infty} 2 e^{-t-T} d t-\int_{T}^{s} e^{3 t-5 T} d t=e^{-T}+2 e^{-2 T}-\frac{e^{-5 T}}{3}\left(e^{3 T}-e^{3 s}\right) \\
& <e^{-T}+\frac{7}{3} e^{-2 T}-\frac{1}{3} e^{3 s-5 T}<\frac{10}{3} e^{-T}-\frac{1}{3} e^{3 s-5 T}=\frac{1}{3} e^{-T}\left(10-e^{3 s-4 T}\right) .
\end{aligned}
$$

For every $s>2$, the maximum of the expression $10 e^{-T}-e^{3 s-5 T}$ with respect to $T \in$ $[0, s]$ is obtained exactly when $10 e^{-T}-5 e^{3 t-5 T}=0$, i.e., when $2 e^{4 T}=e^{3 s}$. Continuing the inequality, we obtain the following for $s \geq T(s>2)$ :

$$
J^{u}(s)<\left.\frac{1}{3} e^{-T}\left(10-e^{3 s-4 T}\right)\right|_{2 e^{4 T-3 s}=1}=\left.\frac{8}{3} e^{-T}\right|_{2 e^{4 T-3 s}=1}=\frac{8 \sqrt[4]{2}}{3} e^{-3 s / 4} .
$$

Thus, for every control that is different from $u^{0} \equiv 0$, we have $J^{u}(s)<$ $\max \left\{4 e^{-3 s / 4}, e^{-s}\right\}$ for all $s>2$; moreover, $\lim _{t \rightarrow \infty} J^{u}(t)=-\infty$. We see that from $x^{0} \equiv$ $0, J^{u^{0}} \equiv 0$ it follow that $u^{0} \equiv 0$ is uniformly overtaking optimal.

Substituting the Hamiltonian $\mathcal{H}=\psi(x+u)+\lambda e^{-2 t} x\left(1-x^{4}\right)$ into relation (4c), we obtain $u^{0} \in \arg \max _{p \in[0,1]} \psi p$, whence by $u^{0} \equiv 0$ we have $\psi^{0}(t) \leq 0$ for all $t \in \mathbb{T}$ for each Lagrange multiplier $\left(\psi^{0}, \lambda^{0}\right) \in \Lambda$. Now, (4b) is equivalent to the equation

$$
\dot{\psi}=-\psi-\lambda e^{-2 t},
$$

all solutions $(\lambda, \psi)$ of which have the form $\left(2 C, C e^{-2 t}+D e^{-t}\right)$. In particular, if a nontrivial Lagrange multiplier $\left(1, \psi^{0}\right)$ associated with $\left(x^{0}, u^{0}\right)$ satisfies transversality condition (20b); then, $\psi^{0}(t) e^{t} \rightarrow 0, D=0$, and $\psi^{0}=e^{-2 t} / 2$. However, $\psi^{0} \leq 0$, therefore, in this example, there is no nontrivial Lagrange multiplier $\left(1, \psi^{0}\right)$ associated with $\left(x^{0}, u^{0}\right)$ that satisfies transversality condition (20b). In particular, a non-degenerate $\tau$ vanishing multiplier does not conform to explicit representation (22c) in this example (see Lemma 2 and Corollary 8).

Consider the solution $\psi^{0}(t)=-e^{-t}+e^{-2 t}$ and $\lambda^{0}=1$. Core relations of Maximum Principle (4a)-(5a) hold for $\left(x^{0}, u^{0}, \lambda^{0}, \psi^{0}\right)$. We claim that $\left(\psi^{0}, \lambda^{0}\right)$ is a $\tau$-vanishing Lagrange multiplier for all $\tau=\left(\tau_{n}\right)_{n \in \mathbb{N}} \uparrow \infty$. Indeed, put $\lambda_{n}=1$. For each $\xi \in \mathbb{R}$, consider the functions given $\psi_{\xi}, x_{\xi}$ by the following relations:

$$
x_{\xi}(t) \triangleq \xi e^{t} \quad \psi_{\xi}(t)=-e^{-t}+e^{-2 t}+\frac{5}{3} \xi^{4} e^{2 t}, \quad \forall t \in \mathbb{T} .
$$

Note that the maps $\xi \mapsto x_{\xi}, \xi \mapsto \psi_{\xi}$ are continuous, in particular, $x_{\xi}$ converges to $x_{0}=x^{0}$, and $\psi_{\xi}$ converges to $\psi_{0}=\psi^{0}$ if $\xi$ tends to zero. Then, every $x_{\xi}$ is a solution of equation ( $8 \mathrm{~b})$. Moreover,

$$
\dot{\psi}_{\xi}=-\psi_{\xi}-e^{-2 t}+5 \xi^{4} e^{2 t}=-\psi_{\xi}-e^{-2 t}\left(1-5 x_{\xi}^{4}\right)=-\frac{\partial \mathcal{H}}{\partial x}\left(x_{\xi}(t), t, u^{0}(t), 1, \psi_{\xi}(t)\right),
$$

and $\left(\psi_{\xi}, x_{\xi}, 1\right)$ satisfies $(8 \mathrm{a})-(8 \mathrm{c})$ for all $\xi \in \mathbb{R}$. Note that there exists a root $\xi(t)$ of equation $-e^{-t}+e^{-2 t}+\frac{5}{3} \xi^{4} e^{2 t}=0$ for all $t \in \mathbb{T}$; moreover, $\xi(t)$ tends to zero as $t \rightarrow$ $\infty$. Consider a sequence $\tau \uparrow \infty$. Put $x_{n} \triangleq x_{\xi\left(\tau_{n}\right)}, \psi_{n} \triangleq \psi_{\xi\left(\tau_{n}\right)}$. Then $\left(\psi^{0}, x^{0}, \lambda^{0}\right)$ is a pointwise limit of the sequence of solutions $\left(\psi_{n}, x_{n}, \lambda_{n}\right)$ of system (8a)-(8c) such that 
$\psi_{n}\left(\tau_{n}\right)=0$ for every $n \in \mathbb{N}$. Thus, $\left(\lambda^{0}, \psi^{0}\right)=\left(1,-e^{-t}+e^{-2 t}\right)$ is $\tau$-vanishing Lagrange multiplier for all $\tau=\left(\tau_{n}\right)_{n \in \mathbb{N}} \uparrow \infty$.

A degenerate vanishing solution exists as well. For each $\xi \in \mathbb{R}$, let us redefine the functions $\psi_{\xi}, x_{\xi}$ by the relations

$$
x_{\xi}(t)=\xi e^{t}, \quad \psi_{\xi}(t)=-e^{-t}+\xi e^{-2 t}+\frac{5}{3} \xi^{5} e^{2 t}, \quad \forall t \in \mathbb{T} .
$$

In addition, $x_{\xi}$ converge to $x_{0}=x^{0}$, and $\psi_{\xi}$ converge to $\psi_{0}=\psi^{0}$ if $\xi$ tends to zero; every $x_{\xi}$ is a solution of equation ( $\left.8 \mathrm{~b}\right)$. Moreover,

$$
\dot{\psi}_{\xi}=-\psi_{\xi}-\xi e^{-2 t}+5 \xi^{5} e^{2 t}=-\psi_{\xi}-\xi e^{-2 t}\left(1-5 \xi^{4} e^{4 t}\right)=-\frac{\partial \mathcal{H}}{\partial x}\left(x_{\xi}(t), t, u^{0}(t), \xi, \psi_{\xi}(t)\right)
$$

and $\left(\psi_{\xi}, x_{\xi}, \xi\right)$ satisfies $(8 \mathrm{a})-(8 \mathrm{c})$ for every $\xi \in[0,1]$. Note that there exists a root $\xi(t)>0$ of equation $-e^{-t}+\xi e^{-2 t}+\frac{5}{3} \xi^{5} e^{2 t}=0$ for all $t \in \mathbb{T}$; moreover $\xi(t)$ tends to zero as $t \rightarrow \infty$. Consider a sequence $\tau \uparrow \infty$. Put $\lambda_{n} \triangleq \xi\left(\tau_{n}\right), x_{n} \triangleq x_{\xi\left(\tau_{n}\right)}, \psi_{n} \triangleq \psi_{\xi\left(\tau_{n}\right)}$. Then, $\left(\psi^{0}, x^{0}, \lambda^{0}\right)$ is the pointwise limit of the sequence of solutions $\left(\psi_{n}, x_{n}, \lambda_{n}\right)$ of system (8a)-(8c) such that $\psi_{n}\left(\tau_{n}\right)=0$ for every $n \in \mathbb{N}$. Thus, $\left(\lambda^{0}, \psi^{0}\right)=\left(0,-e^{-t}\right)$ is a degenerate $\tau$-vanishing Lagrange multiplier for all $\tau=\left(\tau_{n}\right)_{n \in \mathbb{N}} \uparrow \infty$.

We claim that in this example, for each vanishing solution, i.e., for each limit of solutions of problems (8a)-(8c), we have $x_{n} \not \equiv 0$ for all $n \in \mathbb{N}$. Suppose the contrary: let there exist such $\tau$-vanishing solution $\left(\psi^{0}, \lambda^{0}\right)$ for some sequence $\tau \uparrow \infty$. Then, for a sequence of positive numbers $\lambda_{n}, \psi^{0}$ is the limit of some subsequence of solutions $\psi_{n}$ of the Cauchy problems $\dot{\psi}_{n}=-\psi_{n}-\lambda_{n} e^{-2 t}, \psi_{n}\left(\tau_{n}\right)=0$. But for a solution $\psi^{n}$ of such equation the relations $\psi^{n}\left(\tau_{n}\right)=0$ and $\lambda_{n}>0$ imply that $\psi^{n}(t)>0$ for $t<\tau_{n}$. Then, for their pointwise limit $\psi^{0}$, it holds that $\psi^{0} \geq 0$. However, as a vanishing solution, $\psi^{0}=0$ must satisfy ( $\left.4 \mathrm{c}\right)$, i.e., $\psi^{0} \leq 0$. Thus, $\psi^{0} \equiv 0$. But $\psi^{0} \equiv 0$ is a solution of the adjoint system only if $\lambda^{0}=0$. Then, the solution $\left(\lambda^{0}, \psi^{0}\right)$ is trivial and, therefore, cannot be vanishing. This contradiction proves that in this example, there is no vanishing Lagrange multiplier such that its sequence of solutions $\left(x_{n}, \psi_{n}, \lambda_{n}\right)$ of (8a)-(8c) satisfies $x_{n} \equiv x^{0}$.

In [5, Remark 3], a question was formulated: is equality (22c) a necessary condition of optimality if $f, g$ do not decrease by $x$. We have a partial answer to the question from [5, Remark 3]. Example 4 completely satisfies all conditions of [5, Theorem 5], Corollary 7 except one: $g$ only increases in a neighborhood (of a fixed radius) of the optimal trajectory. This fact proved to be sufficient to make it impossible for a nonnegative shadow price to exist in solutions of the Pontryagin Maximum Principle and to negate transversality condition (20b), explicit formula (22c), and inequality (18). Thus, the answer to the question from [5, Remark 3] is negative if the monotonicity holds only in some fixed neighborhood of optimal solution.

Let us show the example of reducing an infinite horizon optimal control problem to the boundary problem.

Example 5 In [7], the following stylized microeconomic problem was considered:

$$
\begin{aligned}
\dot{x}(t) & =-v x(t)+u(t), \quad x(0)=K_{0}, u \geq 0 ; \\
J^{u}(T) & =\int_{0}^{T} e^{-d t}\left[e^{p t}(x(t))^{\sigma}-\frac{b}{2}(u(t))^{2}\right] d t \stackrel{T \rightarrow \infty}{\rightsquigarrow} \max .
\end{aligned}
$$


Here, $u(t)$ is the investment, $v \geq 0$ is the depreciation rate, $K_{0}>0$ is the given initial capital stock, $e^{-d t}$ is the discount factor $(d \geq 0), e^{p t} \geq 0$ is the (exogenous) factor of technological advancement $(p \geq 0), b u^{2}(t)(b>0)$ is the cost of investment $u(t)$, and $\sigma \in(0,1]$ defines the production function. Under the assumption $d+v>$ $\frac{p}{2-\sigma}$, it is shown that there are no optimal solutions for $p>d+v$, and, for $p<d+v$, each locally weakly overtaking control induces a solution of the boundary problem (see [7]).

Consider the following objective functional:

$$
J^{u}(T)=\int_{0}^{T} g(t)(x(t))^{\sigma}-h(t) \frac{b}{2}(u(t))^{2} d t \stackrel{T \rightarrow \infty}{\sim} \max .
$$

Here, $h(t)$ is the discount factor, $g(t)$ is the product of the discount factor and the factor of technological advancement. Suppose that $g(t) \geq 0, h(t)>0$ for a.a. $t \in \mathbb{T}$.

Suppose the locally bounded function $u^{0}$ is a weakly overtaking optimal control $u^{0}$. Then, for some sequence $\tau \uparrow \infty$, this control is $\tau$-optimal. Put $U(t)=\left[0, u^{0}(t)+1\right]$. Then, $u^{0}$ is $\tau$-optimal even under additional constraints $u \in U(t)$. Hence, there exists a $\tau$-vanishing multiplier $\left(\lambda^{0}, \psi^{0}\right) \in \Lambda$.

Now, for all $\xi \in \mathbb{X}$, we have $A_{\xi}=e^{-v t}$,

$$
I_{\xi}(T)=\int_{0}^{T} g(t) \sigma x_{\xi}^{\sigma-1}(t) e^{-v t} d t=\sigma \int_{0}^{T} g(t) e^{-v t} x_{\xi}^{\sigma-1}(t) d t
$$

Note that $x_{\xi}(t)-x^{0}(t)=\xi e^{-v t} ;$ now we have

$$
I_{\xi}(T)-I_{0}(T)=\sigma \int_{0}^{T} g(t) e^{-\sigma v t}\left[\left(x^{0}(t) e^{v t}+\xi\right)^{\sigma-1}-\left(x^{0}(t) e^{v t}\right)^{\sigma-1}\right] d t
$$

It is easy see that $\left|(r+\xi)^{\sigma-1}-r^{\sigma-1}\right| \leq\left(2^{2-\sigma}-2\right)|\xi| r^{\sigma-2} \leq\left(2^{2-\sigma}-2\right) K_{0}|\xi| r^{\sigma-1}$ if $2|\xi|<K_{0} \leq r$. Since the function $x^{0}(t) e^{v t}$ is monotonically increasing, we obtain

$$
\left|I_{\xi}(T)-I_{0}(T)\right| \leq\left|\int_{0}^{T} g(t) e^{-v t}\left(x^{0}\right)^{\sigma-1} d t\right|\left(2^{2-\sigma}-2\right) K_{0}|\xi|=\left|I_{0}(T)\right|\left(2^{2-\sigma}-2\right) K_{0}|\xi|
$$

for all $T \in \mathbb{T}, 2|\xi|<K_{0}$. Now, by Corollary 9, considering the subsequence if necessary, we have the conclusion of Theorem 3. In particular, since the functions $g, x$ are nonnegative, we see that the functions $I_{\xi}, I_{*}$ is nonnegative.

We claim that $\left(I_{0}\left(\tau_{n}\right)\right)_{n \in \mathbb{N}}$ is bounded. Assume the converse; then, considering the subsequence if necessary, we come to (21b) and (22b), whence $\lim _{\xi \rightarrow 0, n \rightarrow \infty} I_{\xi}\left(\tau_{n}\right)=$ $+\infty$, now $\iota^{*}=1, \lambda^{0}=0$ and by $(4 \mathrm{c})$ we have

$$
u^{0}(t)=\arg \max _{u \in U(t)} e^{v t} I_{0}(t)(u-v x)=\arg \max _{u \in\left[0, u^{0}(t)+1\right]} I_{0}(t) u=u^{0}(t)+1,
$$

which is impossible. This contradiction proves the boundedness of sequence $\left(I_{0}\left(\tau_{n}\right)\right)_{n \in \mathbb{N}}$. 
Now, there exists a finite limit $I_{*}$ of $\left(I_{0}\left(\tau_{n}^{\prime}\right)\right)_{n \in \mathbb{N}}$ for some $\tau^{\prime} \subset \tau$. Since the functions $g, x$ are nonnegative, $I_{0}(t)$ converges to $I_{*}$ as $t \rightarrow \infty$. By Theorem 3, we have (22a); i.e., $\lambda^{0}=1, \psi^{0}(T)=\left(I_{*}-I_{0}(t)\right) e^{\nu t}$,

$$
\begin{aligned}
u^{0}(t) & =\arg \max _{u \in\left[0, u^{0}(t)+1\right]} e^{\nu t}\left(I_{*}-I_{0}(t)\right)(-v x+u)+g(t)\left(x^{0}(t)\right)^{\sigma}-h(t) \frac{b}{2} u^{2} \\
& =\arg \max _{u \in\left[0, u^{0}(t)+1\right]} e^{\nu t}\left(I_{*}-I_{0}(t)\right) u-h(t) \frac{b}{2} u^{2}=\frac{e^{\nu t}}{b h(t)}\left(I_{*}-I_{0}(t)\right) \quad \text { for a.a.t } \in \mathbb{T} .
\end{aligned}
$$

Consider $I(t) \triangleq I^{*}-I_{0}(t)$; differentiating $I(t)$ with respect to $t$, we finally close (4a)(4b) into the boundary problem

$$
\begin{aligned}
\dot{x}^{0} & =-v x^{0}+\frac{e^{v t}}{b h(t)} I, \quad x^{0}(0)=K_{0}, \\
\dot{I} & =-\sigma g(t) e^{-v t}\left(x^{0}\right)^{\sigma-1}, \\
I(t) & =I^{*}-I_{0}(t) \rightarrow 0 \text { as } n \rightarrow \infty .
\end{aligned}
$$

Each $\tau$-optimal control (weakly overtaking optimal control) generates the unique solution of this boundary problem (28a)-(28c). For $\sigma=1$ if such solution exists; there also exists a finite $\int_{0}^{\infty} e^{-v t} g(t) d t$.

It is possible to find the explicit solution of boundary problem (28a)-(28c) in some specific cases. For example, let the discount factor equal $\frac{1}{(1+t)^{4 / 3}}$, let the factor of technological advancement be equal to 1 . For

$$
g(t)=h(t)=\frac{1}{(1+t)^{4 / 3}}, v=0, \sigma=1 / 2, b=\frac{3}{8}, K_{0}=1
$$

we have

$$
x^{0}(t)=(1+t)^{4 / 3}, u^{0}(t)=\frac{4}{3}(1+t)^{1 / 3}, I(t)=\frac{1}{2(1+t)}, J^{u^{0}}(t)=(1+t)^{2 / 3} .
$$

The discount factor $g(t)=\frac{1}{(1+t)^{4 / 3}}$ here is not arbitrary, its power $\alpha=3,96 / 2,94 \approx$ $4 / 3$ was determined by means of statistic analysis in [46]. A thorough discussion of various discount functions and their properties could be found in [20, 21, 47]. These papers do not generally assume the discount function to be dominated by a decreasing exponential function and do not assume its monotonicity.

Acknowledgements I am grateful to an anonymous referee for helpful comments. I would like to express my gratitude to S. M. Aseev, A. G. Chentsov, A. M. Tarasyev, N. Yu. Lukoyanov, and Yu. V. Averboukh for valuable discussion in course of writing this article. Special thanks to Ya. V. Salii for the translation. This study was supported by the Russian Foundation for Basic Research (RFBR) under grants nos. 12-01-31172, 13-01-90414, and 12-01-00537.

\section{Appendix}

Proof of Proposition 2 For all $n \in \mathbb{N}$, consider a set $\bar{G}_{n} \triangleq\left\{(t, y(t)) \mid \forall y \in \widetilde{\mathfrak{A}}\left[\widetilde{u}^{0}\right], t \in\right.$ $[0, n]\}$; by the extendability condition for $a$, this set is bounded. Therefore, on this set, the function $a\left(t, y, u^{0}(t)\right)$ is Lipshitz continuous with respect to $y$ for the 
certain Lipshitz constant $L_{n} \triangleq L_{\bar{G}_{n}}^{a} \in \mathcal{L}_{l o c}^{1}(\mathbb{T}, \mathbb{T})$. For all $t \in[0, n]$, define $M_{n}(t) \triangleq$ $\int_{0}^{t} L_{n}(\tau) d \tau$.

Fix $n \in \mathbb{N}$; for all $t \in[n-1, n), u \in \mathbb{U}$, consider the numbers

$$
R(t, u) \triangleq \sup _{y \in \bar{G}_{n}}\left\|a(t, y, u)-a\left(t, y, u^{0}(t)\right)\right\|_{E}, \quad w^{0}(t, u) \triangleq\left\|u-u^{0}(t)\right\|+e^{M_{n}(t)} R(t, u) .
$$

We define the functions $R, w^{0}$ on the whole $\mathbb{T} \times \mathbb{U}$. In [31, Lemma 7.1], the author proved that these functions are Carathéodory functions, and $w^{0} \in(N u l l)\left(u^{0}\right)$ is the required weight.

Proof of Proposition 3 For every $n \in \mathbb{N}$, let us consider the problem

$$
J^{\eta}\left(\tau_{n}\right)-\gamma_{n} \mathfrak{L}_{w}[\eta]\left(\tau_{n}\right)=\int_{0}^{\tau_{n}} \int_{U(t)} g\left(t, x^{\eta}(t), u\right) d \eta(t) d t-\gamma_{n} \mathfrak{L}_{w}[\eta]\left(\tau_{n}\right) \rightarrow \max .
$$

Here, the functional is bounded from above by the number $J^{u^{0}}\left(\tau_{n}\right)+\gamma_{n}^{2}$, therefore, it has the supremum. Every summand continuously depends on $\eta$, which covers the compact $\widetilde{\mathfrak{U}}$; therefore, there is an optimal solution for this problem in $\widetilde{\mathfrak{U}}$; let us denote one of them by $\eta^{n}$, and its trajectory by $x^{n}$.

For every $\gamma \in \mathbb{T}$ let the function $\mathcal{H}_{\gamma}: \mathbb{X} \times \mathbb{T} \times \mathbb{U} \times \mathbb{T} \times \mathbb{X} \rightarrow \mathbb{R}$ be given by

$$
\mathcal{H}_{\gamma}(x, t, u, \lambda, \psi) \triangleq \mathcal{H}(x, t, u, \lambda, \psi)-\gamma w(t, u) .
$$

Then, by the Maximum Principle [15, Theorem 5.2.1], there exists $\left(\lambda^{n}, \psi^{n}\right) \in(0,1] \times$ $C\left(\left[0, \tau_{n}\right], \mathbb{X}\right)$ such that relation (5a) and the transversality condition $\psi^{n}\left(\tau_{n}\right)=0$ hold, and

$$
\begin{aligned}
\sup _{p \in U(t)} \mathcal{H}_{\gamma_{n}}\left(x^{n}(t), t, p, \lambda^{n}, \psi^{n}(t)\right) & =\int_{U(t)} \mathcal{H}_{\gamma_{n}}\left(x^{n}(t), t, u, \lambda^{n}, \psi^{n}(t)\right) d \eta^{n}(t), \\
\dot{\psi}^{n}(t) & =-\int_{U(t)} \frac{\partial \mathcal{H}_{\gamma_{n}}}{\partial x}\left(x^{n}(t), t, u, \lambda^{n}, \psi^{n}(t)\right) d \eta^{n}(t)
\end{aligned}
$$

also hold for a.a. $t \in\left[0, \tau_{n}\right]$.

Let us extend the $\left(x^{n}, \eta^{n}, \lambda^{n}, \psi^{n}\right)$ to $\left[\tau_{n}, \infty\right)$ by the generalized control $\left.\widetilde{u}^{0}\right|_{\left[\tau_{n}, \infty\right)}$. Let us denote by $\mathfrak{Z}^{n}$ the set of $(x, \eta, \lambda, \psi)$ that satisfy relations $(5 a), \widetilde{(4 a)}-(\widetilde{4 b})$ a. e. on $\mathbb{T}$, satisfy relation (30a) a. e. on $\left[0, \tau_{n}\right)$, and possess the property $\left.\widetilde{u}^{0}\right|_{\left[\tau_{n}, \infty\right)}=\left.\eta^{n}\right|_{\left[\tau_{n}, \infty\right)}$. Now we have $\left(x^{n}, \eta^{n}, \lambda^{n}, \psi^{n}\right) \in \mathfrak{Z}^{n}$ for every $n \in \mathbb{N}$.

Let us note that all $\mathfrak{Z}^{n}$ are closed and, since these sets are contained in the compact $\widetilde{\mathfrak{Y}}$, these sets are also compact. Hence, the sequence $\left(x^{n}, \eta^{n}, \lambda^{n}, \psi^{n}\right)_{n \in \mathbb{N}}$ has the limit point $\left(x^{00}, \eta^{0}, \lambda^{0}, \psi^{0}\right) \in \widetilde{\mathfrak{Y}}$. Considering, if need be, the subsequence, we can assume that it is the limit of the sequence itself.

For all $t, \gamma, \lambda \in \mathbb{T},(x, \psi) \in \mathbb{X} \times \mathbb{X}$, denote by $\mathcal{P}_{\gamma, \lambda}(t ; x, \psi)$ the set of $p \in U(t)$ that realize the maximum of $\mathcal{H}_{\gamma}(x, t, p, \lambda, \psi)$. For all $\gamma, \lambda \in \mathbb{T},(x, \psi) \in \mathbb{X} \times \mathbb{X}$, the compact-valued map $t \mapsto \mathcal{P}_{\gamma, \lambda}(t ; x, \psi)$ has a measurable selector by virtue of [16, Theorem 3.7]. Then, by [43, Lemm 2.3.11], for an arbitrary function $(x, \psi) \in$ $C(\mathbb{T}, \mathbb{X} \times \mathbb{X})$ the map $t \mapsto \mathcal{P}_{\gamma, \lambda}(t ;(x, \psi)(t))$ also has a measurable selector. Note that relation (30a) also depends on $x, \psi$ and on the parameters $\gamma$ and $\lambda$ upper semicontinuously; moreover, all the relations are integrally bounded on bounded sets. Therefore, by virtue of [43, Theorem 3.5.6], on each finite interval for the funnels 
of solutions of (4a)-(4b), (30a), it is upper semicontinuous in $\gamma, \lambda$. In particular, since $\gamma_{n} \rightarrow 0$ and $\lambda^{n} \rightarrow \lambda^{0}$, the upper limit of the compacts $\mathfrak{Z}^{n}$ is included in $\widetilde{\mathfrak{Z}}$. Hence, $\left(x^{00}, \eta^{0}, \lambda^{0}, \psi^{0}\right) \in \widetilde{\mathfrak{Z}}$.

On the other side, by $w \in(N u l l)\left(u^{0}\right)$ and by optimality of $\eta^{n}, u^{0}$ for their problems, we obtain

$$
\widetilde{J}^{\eta^{n}}\left(\tau_{n}\right)-\gamma_{n} \mathfrak{L}_{w}\left[\eta^{n}\right]\left(\tau_{n}\right) \geq J^{u^{0}}\left(\tau_{n}\right) \stackrel{\widetilde{(2)}}{\geq} \widetilde{J}^{\eta^{n}}\left(\tau_{n}\right)-\gamma_{n}^{2}
$$

therefore, we have $\gamma_{n} \mathfrak{L}_{w}\left[\eta^{n}\right]\left(\tau_{n}\right) \leq \gamma_{n}^{2}$. By $\left.\widetilde{u}^{0}\right|_{\left[\tau_{n}, \infty\right)}=\left.\eta^{n}\right|_{\left[\tau_{n}, \infty\right)}$, we obtain

$$
\mathfrak{L}_{w}\left[\eta^{n}\right](t) \leq \gamma_{n} \quad \forall t \in \mathbb{T} .
$$

For each $t \in \mathbb{T}$, passing to the limit as $n \rightarrow \infty$, we obtain that $\mathfrak{L}_{w}\left[\eta^{0}\right] \leq 0$; i.e., $\mathfrak{L}_{w}\left[\eta^{0}\right](t)=0$ for all $t \in \mathbb{T}$. Since $w \in(N u l l)\left(u^{0}\right)$, we have $\eta^{0}=\widetilde{u}^{0}$ a.e. on $\mathbb{T}$, hence $x^{00} \equiv x^{0}$ and $\left(x^{0}, u^{0}, \lambda^{0}, \psi^{0}\right) \in \mathfrak{Z}$. Moreover, from (30b), we have $\left\|\mathfrak{L}_{w}\left[\eta^{n}\right]\right\|_{C} \rightarrow 0$.

\section{References}

1. Aseev SM, Kryazhimskii AV. The Pontryagin Maximum Principle and transversality conditions for a class of optimal control problems with infinite time horizons. SIAM J Control Optim. 2004;43:1094-119.

2. Aseev SM, Kryazhimskii AV. The Pontryagin Maximum Principle for an optimal control problem with a functional specified by an improper integral. Dokl Math. 2004;69(1):89-91.

3. Aseev SM, Kryazhimskii AV. The Pontryagin Maximum Principle and problems of optimal economic growth. Proc Steklov Inst Math. 2007;257(1):1-255.

4. Aseev SM, Kryazhimskii AV. Shadow prices in infinite-horizon optimal control problems with dominating discounts. Appl Math Comput. 2008;204(2):519-31.

5. Aseev SM, Kryazhimskii AV, Besov KO. Infinite-horizon optimal control problems in economics. Russ Math Surv. 2012;67(2):195-253.

6. Aseev SM, Kryazhimskii AV, Tarasyev AM. The Pontryagin Maximum Principle and transversality conditions for an optimal control problem with infinite time interval. Proc Steklov Inst Math. 2001;233:64-80.

7. Aseev SM, Veliov VM. Maximum Principle for infinite-horizon optimal control; problems with dominating discount. Dyn Contin Discret I. 2012;19(1-2b):43-63.

8. Aseev SM, Veliov VM. Needle variations in infinite-horizon optimal control. IIASA Interim Rept. IR-2012-04; 2012.

9. Aubin JP, Clarke FH. Shadow prices and duality for a class of optimal control problems. SIAM J Control Optim. 1979;17:567-86.

10. Balder EJ. An existence result for optimal economic growth problems. J Math Anal. 1983;95(1):195-213.

11. Benveniste LM, Scheinkman JA. Duality theory for dynamic optimization models of economics: the continuous time case. J Econ Theory. 1982;27:1-19.

12. Carlson DA. Uniformly overtaking and weakly overtaking optimal solutions in infinite-horizon optimal control: when optimal solutions are agreeable. J Optim Theory Appl. 1990;64(1): 55-69.

13. Carlson DA. Nonconvex and relaxed infinite-horizon optimal control problems. J Optim Theory Appl. 1993;78(3):465-91.

14. Carlson DA, Haurie AB, Leizarowitz A. Infinite horizon optimal control. Deterministic and stochastic systems. Berlin: Springer; 1991.

15. Clarke FH. Optimization and nonsmooth analysis. New York: Wiley; 1983.

16. Daniel HW. Survey of measurable selection theorems: an update. In: Lect Notes Math. vol. 794, Springer; 1980. p. 176-219. 
17. Davidson R, Harris R. Nonconvexities in continuous-time investment theory. Rev Econ Stud. 1981;43:235-253.

18. Di Marco SC, Gonzalez RL. Relaxation of minimax optimal control problems with infinite horizon. J Optim Theory Appl. 1999;101(2):285-307.

19. Dmitruk AV, Kuz'kina NV. An existence theorem in an optimal control problem on an infinite time interval. Math Notes. 2005;78(3-4):466-80.

20. Ekeland I. Discounting the future: the case of climate change. Cahiers de la Chaire Finance et Developpement Durable. vol. 27; 2009.

21. Ekeland I, Pirvu TA. Investment and consumption without commitment. Math Finan Econ. 2008;2:57-86.

22. Gamkrelidze RV. Principles of optimal control theory. New York: Plenum Press; 1978.

23. Halkin H. Necessary conditions for optimal control problems with infinite horizons. Econometrica. 1974;42:267-72.

24. Hammond PJ, Kennan J. Uniformly optimal infinite-horizon plans. Int Econ Rev. 1979;20:28396.

25. Kamihigashi T. Necessity of transversality conditions for infinite horizon problems. Econometrica. 2001;69:995-1012.

26. Khan MA, Zaslavski AJ. On locally optimal programs in the Robinson-Solow-Srinivasan model. J Econ. 2010;99:65-92.

27. Khlopin DV. On extension of conflict control problems on infinite horizon (in Russian). Bull Udmurt Univ. Mathematics, Mechanics, Computer Science; 2011. vol. 1, p. 105-12.

28. Khlopin DV. Necessity of transversality conditions for infinite horizon problems and stability of shadow prices (in Russian). Tambov University Report; 2011. vol. 16(4), p. 1209.

29. Khlopin, DV. Necessary conditions of optimality for infinite-horizon control problems without discount factor. In: 12th Viennese workshop optimal control, dynamic games and nonlinear dynamics (ORCOS-2012); 30 May-2 June 2012. p. 45.

30. Khlopin DV. On necessary boundary conditions for strongly optimal control in infinite horizon control problems (in Russian). Bull. Udmurt. Univ. Mathematics, Mechanics, Computer Science; 2013. vol. 1, p. 49-58.

31. Khlopin DV. Necessary conditions of overtaking equilibrium for infinite horizon (in Russian). Mat Teor Igr Prilozh. 2013;2(5):105-136.

32. Michel P. On the transversality condition in infinite horizon optimal problems. Econometrica. 1982;50:975-84.

33. Pickenhain S. On adequate transversality conditions for infinite horizon optimal control problems - a famous example of Halkin. In: Crespo Cuaresma J, Palokangas T, Tarasyev A, editors. Dynamic systems, economic growth, and the environment. Berlin etc.: Springer; 2010 (Dynamic Modeling and Econometrics in Economics and Finance 12). p. 3-22.

34. Pontryagin LS et al. The mathematical theory of optimal processes. Moscow: Fizmatgiz; 1961.

35. Sagara N. Value functions and transversality conditions for infinite-horizon optimal control problems. Set-Valued Var Anal. 2010;18(1):1-28.

36. Seierstad A. Necessary conditions for nonsmooth, infinite-horizon optimal control problems. J Optim Theory Appl. 1999;103(1):201-30.

37. Seierstad A. Fields of extremals and infinite horizon optimal control problems. Optim Control Appl Meth. 1998;19:377-92.

38. Seierstad A, Sydsæter K. Optimal control theory with economic applications. Amsterdam: North-Holland; 1987.

39. Shell K. Applications of Pontryagin's maximum principle to economics. In: Mathematical systems theory and economics 1. Lect. Notes Oper. Res. Math. Econ. vol. 11. Berlin: Springer; 1969. p. 241-92.

40. Smirnov GV. Transversality condition for infinite-horizon problems. J Optim Theory Appl. 1996;88(3):671-88.

41. Stern LE. Criteria of optimality in the infinite-time optimal control problem. J Optim Theory Appl. 1984;44(3):497-508.

42. Tan H, Rugh WJ. Nonlinear overtaking optimal control: sufficiency, stability, and approximation. IEEE Trans Autom Control. 1998;43(12):1703-18.

43. Tolstonogov AA. Differential inclusions in a Banach space. Mathematics and its applications. vol. 524. Dordrecht: Kluwer Academic Publishers; 2000.

44. Warga J. Optimal control of differential and functional equations. New York: Academic Press; 1972. 
45. Weber T. An infinite-horizon maximum principle with bounds on the adjoint variable. J Econ Dyn Control. 2006;30:229-41.

46. Weitzman M. Gamma discounting. Am Econ Rev. 2001;91(1):260-71.

47. Weitzman M. Risk-adjusted gamma discounting. J Environ Econ Manag. 2010;60:1-13.

48. Ye JJ. Nonsmooth Maximum Principle for infinite-horizon problems. J Optim Theory Appl. 1993;76:485-500.

49. Zaslavski AJ. Turnpike properties in the calculus of variations and optimal control. New York: Springer; 2006 